\title{
Late Quaternary Deglaciation, Glaciomarine Sedimentation and Glacioisostatic Recovery in the Rivière Nastapoka Area, Eastern Hudson Bay, Northern Québec \\ Déglaciation, sédimentation glaciomarine et relèvement glacio-isostatique au Quaternaire supérieur dans la région de la rivière Nastapoka, à l'est de la baie d'Hudson (Québec nordique) Deshielo cuaternario tardío, sedimentación glaciomarina y registro glacioisostático en el área del rivière Nastapoka, al este de la bahía de Hudson en el norte de Québec
}

Patrick Lajeunesse et Michel Allard

Volume 57, numéro 1, 2003

URI : https://id.erudit.org/iderudit/010331ar

DOI : https://doi.org/10.7202/010331ar

Aller au sommaire du numéro

Éditeur(s)

Les Presses de l'Université de Montréal

ISSN

0705-7199 (imprimé)

1492-143X (numérique)

Découvrir la revue

Citer cet article

Lajeunesse, P. \& Allard, M. (2003). Late Quaternary Deglaciation, Glaciomarine Sedimentation and Glacioisostatic Recovery in the Rivière Nastapoka Area, Eastern Hudson Bay, Northern Québec. Géographie physique et Quaternaire, 57(1), 65-83. https://doi.org/10.7202/010331ar
Résumé de l'article

Dans la région de la rivière Nastapoka, le retrait de l’inlandsis du Québec-Labrador sous l'influence de la topographie s'est déroulé en quatre phases. Les datations au radiocarbone montrent que la déglaciation a débuté vers 8,3 ka étal. BP et qu'elle a été caractérisée par une stabilisation du front glaciaire dans les collines Nastapoka ayant mené à la mise en place d'une ceinture de complexes sédimentaires alors que le niveau marin relatif était élevé (phase 1). Par la suite, le front glaciaire a rapidement reculé vers l'est dans une zone au relief peu accidenté en déposant un épais couvert de silt argileux dans les dépressions rocheuses ; le niveau marin relatif était encore élevé (phase 2). Le front glaciaire s'est ensuite de nouveau stabilisé jusqu'à au moins $7.2 \mathrm{ka}$ étal. BP dans une région au relief plus accidenté sur le plateau précambrien, au-delà de la limite orientale de l'invasion de la Mer de Tyrrell (phase 3). Cette pause a mené à la mise en place d'une ceinture de deltas fluvio-glaciaires alors que le niveau marin relatif était plus bas. Puis, avec le retrait complet du front glaciaire de la région et l'ablation de l'inlandsis au centre du Québec-Labrador, les eaux de fonte ont transporté d'importantes quantités de sédiments glaciaires selon des processus fluviaux et l'incision en terrasses des dépôts fluvio-glaciaires et marins déposés antérieurement (phase 4). Le relèvement glacio-isostatique de la région a atteint des taux de $0,07 \mathrm{~m} / \mathrm{an}$ au début de la déglaciation, puis a diminué pour atteindre $0,04 \mathrm{~m} / \mathrm{an}$ entre 6 et 5 ka étal. BP et $0,016 \mathrm{~m} / \mathrm{an}$ au cours des 1000 dernières années. 


\section{LATE QUATERNARY DEGLACIATION, GLACIOMARINE SEDIMENTATION AND GLACIOISOSTATIC RECOVERY IN THE RIVIĖRE NASTAPOKA AREA, EASTERN HUDSON BAY, NORTHERN QUÉBEC}

Patrick LAJEUNESSE* and Michel ALLARD, Centre d'études nordiques and Département de géographie, Université Laval, Québec, Québec G1K 7P4.

ABSTRACT This study presents a paleoenvironmental reconstruction of deglaciation dynamics and chronology, glaciomarine and postglacial sedimentation, as well as glacioisostatic recovery in the Rivière Nastapoka area, eastern Hudson Bay. Results indicate that the retreat of Québec-Labrador ice was mainly controlled by topography and was marked by four phases. Radiocarbon dates indicate that deglaciation began about $8.3 \mathrm{ka}$ cal. BP and was characterized by a stillstand of the ice margin in the Nastapoka Hills that lead to the deposition of a drift belt in a high relative sealevel (Phase 1). After this stabilisation, the ice margin retreated rapidly eastward in a region of low relief and deposited a drape of silty clay in a falling relative sea-level (Phase 2). A second phase of stabilization of the ice margin lasted until at least $7.2 \mathrm{ka}$ cal.BP on the higher shield peneplaine east of the limit of the Tyrrell Sea (Phase 3). This lead to the deposition of a belt of glaciofluvial deltas in a lower relative sea-level. Following this stillstand, the eastward retreat and subsequent ablation of the ice in central Québec-Labrador generated meltwater that transported large volumes of glacial sediments by fluvial processes and downcutting of fluvial terraces in previously deposited glaciofluvial and marine sediments (Phase 4). Glacioisostatic rebound reached $0.07 \mathrm{~m} / \mathrm{yr}$ during the early phase of deglaciation and decreased to $0.04 \mathrm{~m} / \mathrm{yr}$ between 6 and $5 \mathrm{ka} \mathrm{cal}$. BP and $0.016 \mathrm{~m} / \mathrm{yr}$ in the last 1000 years.
RÉSUMÉ Déglaciation, sédimentation glaciomarine et relèvement glacio-isostatique au Quaternaire supérieur dans la région de la rivière Nastapoka, à l'est de la baie d'Hudson (Québec nordique). Dans la région de la rivière Nastapoka, le retrait de l'inlandsis du QuébecLabrador sous l'influence de la topographie s'est déroulé en quatre phases. Les datations au radiocarbone montrent que la déglaciation a débuté vers 8,3 ka étal. BP et qu'elle a été caractérisée par une stabilisation du front glaciaire dans les collines Nastapoka ayant mené à la mise en place d'une ceinture de complexes sédimentaires alors que le niveau marin relatif était élevé (phase 1). Par la suite, le front glaciaire a rapidement reculé vers l'est dans une zone au relief peu accidenté en déposant un épais couvert de silt argileux dans les dépressions rocheuses; le niveau marin relatif était encore élevé (phase 2). Le front glaciaire s'est ensuite de nouveau stabilisé jusqu'à au moins 7.2 ka étal. BP dans une région au relief plus accidenté sur le plateau précambrien, audelà de la limite orientale de l'invasion de la Mer de Tyrrell (phase 3). Cette pause a mené à la mise en place d'une ceinture de deltas fluvio-glaciaires alors que le niveau marin relatif était plus bas. Puis, avec le retrait complet du front glaciaire de la région et l'ablation de l'inlandsis au centre du Québec-Labrador, les eaux de fonte ont transporté d'importantes quantités de sédiments glaciaires selon des processus fluviaux et l'incision en terrasses des dépôts fluvio-glaciaires et marins déposés antérieurement (phase 4). Le relèvement glacio-isostatique de la région a atteint des taux de $0,07 \mathrm{~m} / \mathrm{an}$ au début de la déglaciation, puis a diminué pour atteindre $0,04 \mathrm{~m} / \mathrm{an}$ entre 6 et $5 \mathrm{ka}$ étal. BP et $0,016 \mathrm{~m} / \mathrm{an}$ au cours des 1000 dernières années.
RESUMEN Deshielo cuaternario tardío, sedimentación glaciomarina y registro glacioisostático en el área del rivière Nastapoka, al este de la bahía de Hudson en el norte de Québec. En la región del rivière Nastapoka el retiro del inlandsis Québec-Labrador bajo la influencia de la topografía, se efectuó en cuatro etapas. La datación con radiocarbono muestra que el deshielo dio inicio hace unos 8300 años. Este se caracterizó por una estabilización del frente glaciar en las colinas Nastapoka que llevó al establecimiento de un cinturón de complejos sedimentarios mientras que el nivel relativo del mar era elevado (etapa 1). Más tarde el frente glaciar retrocedió rápidamente hacia el este en una zona poco accidentada depositando una gruesa capa de arcilla en las depresiones rocosas; una vez mas cuando el nivel relativo del mar era aun elevado (etapa 2). Posteriormente, hace unos 7200 años el frente glaciar fue nuevamente estable dando origen a un relieve más accidentado sobre la meseta precámbrica mas allá del límite oriental de la región cubierta por el mar de Tyrell (etapa 3). Esta pausa llevó al establecimiento de un conjunto de deltas fluvioglaciares caracterizado por un nivel relativo del mar bajo. Luego, con el retiro completo del frente glaciar de la zona de ablación del inlandsis al centro de Québec-Labrador, las aguas de fusión transportaron cantidades importantes de sedimentos glaciares mediante procesos fluviales de incisión sobre las terrazas de depósitos fluvioglaciares y marinos depositados con anterioridad (etapa 4). Al inicio del deshielo, hace unos 6000 a 5000 años, el elevamiento glacioisostático de la región alcanzó una taza de $0,07 \mathrm{~m}$ al año, más tarde disminuyó hasta alcanzar $0,04 \mathrm{~m}$ por año y finalmente en el transcurso de los últimos 1000 años llego a 0,016 m por año. 


\section{INTRODUCTION}

Late Quaternary glacial geology of Hudson Bay has been relatively well described from research carried out at sea using marine geophysical methods supported by a few cores (Josenhans et al., 1988; Bilodeau et al., 1990; Josenhans and Zevenhuizen, 1990; Gonthier et al., 1993; Zevenhuizen, 1996; Hill et al., 1999). However, in the terrestrial coastal sectors of eastern Hudson Bay, only a small number of papers (HillaireMarcel, 1976; Hillaire-Marcel et al., 1981; Allard and Seguin, 1985; Parent and Paradis, 1994; Parent et al., 1995) have addressed the regional history of ice retreat. Recently, Lajeunesse and Allard $(2002,2003)$ reported on the presence of a series of emerged ice-proximal glaciomarine fans that were deposited during a short period of stabilization of the ice margin in the Rivière Nastapoka area in association with the first phase of deglaciation of southeastern Hudson Bay. The regional associations of these submarine fans with other glacial landforms, local deglaciation dynamics and the rapid glacioisostatic rebound remain nevertheless poorly described in this sector of Hudson Bay. The influence of basin topography on ice retreat and glaciomarine sediment deposition dynamics, which has been well demonstrated in other regions of the world (e.g., Meier and Post, 1987; Stewart, 1991;
Seramur et al., 1997; Ó Cofaigh, 1998), remains unexamined in regards to the deglacial marine transgression in the region (Tyrrell Sea).

In this paper, we describe from on-land evidence the geomorphic, sedimentary, stratigraphic and chronologic record of Late Quaternary ice recession, marine transgression, sedimentation, and glacioisostatic rebound along a stretch of coast located between the northern tip of Lac Guillaume-Delisle and Rivière Richard, eastern Hudson Bay. This integrated approach leads to point out the mechanisms and chronology by which deglaciation and glaciomarine sedimentation occurred in the context of a falling relative sea-level (RSL) due to glacioisostatic recovery.

\section{STUDY AREA}

The study area is located between $56^{\circ}-57^{\circ} \mathrm{N}$ and $76^{\circ} 40^{\prime}-$ $75^{\circ} 30^{\prime} \mathrm{W}$, on the eastern coast of Hudson Bay (Fig. 1). Bedrock geology of most of the study area consists of Archean granitic-gneiss (Stevenson, 1968; Avramtchev, 1982). In the southeast sector of the study area, important outcrops of Proterozoic volcano-sedimentary rocks such as basalt, quartzite and dolomite make up high cuesta ridges that face

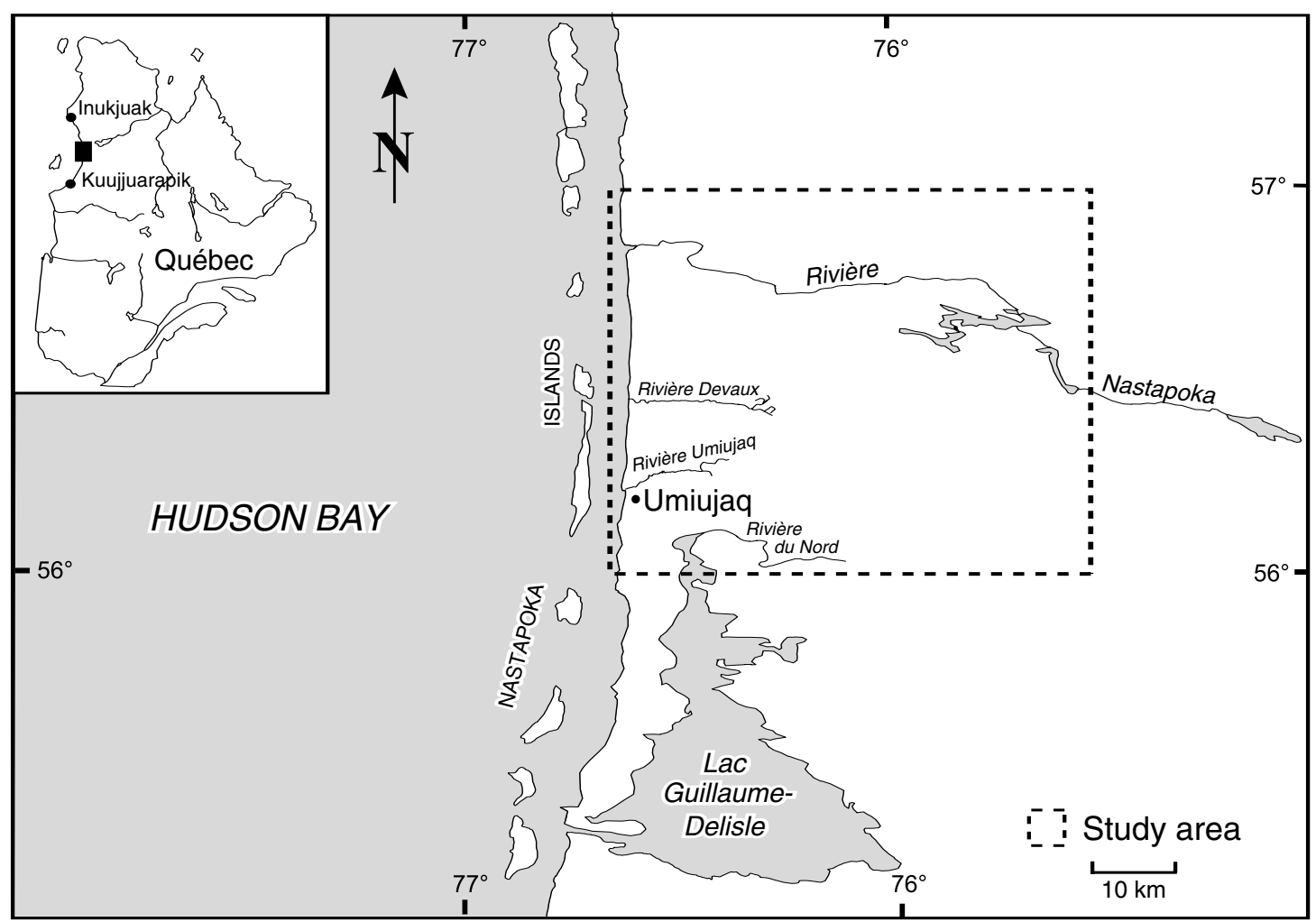

FIGURE 1. Location map of the Rivière Nastapoka and of the study

Carte de localisation de la rivière Nastapoka et de la région d'étude, area, eastern Hudson Bay. sur la côte est de la baie d'Hudson. 
east and enclose Lac Guillaume-Delisle. The area has four physiographic sectors: 1) the coastal slope, which is a narrow, 4-7 km wide, coastal fringe from the actual seashore to the foot of the hills, following a gently inclined $<3^{\circ}$ slope; 2 ) the Nastapoka Hills, a hill range parallel to the coast located between $\sim 4-14 \mathrm{~km}$ inland reaching mean elevations of 280-320 $\mathrm{m}$ above sea level (asl) and maximum elevations of $420 \mathrm{~m}$ asl; 3) an inner basin, i.e. an area of lower and less rugged topography with elevations generally $<200 \mathrm{~m}$ asl and located between 14-23 km from the seashore; and 4) the Hudsonian Plateau, a peneplain with elevations varying between 200-300 m asl extending from $\sim 23 \mathrm{~km}$ inland to well beyond the eastern limit of the study area. The most important river, Rivière Nastapoka, has two waterfalls along its course within the study area. The first falls are located $1 \mathrm{~km}$ from the shore and create a drop of $>40 \mathrm{~m}$. The second falls are located $7 \mathrm{~km}$ inland and are $46 \mathrm{~m}$ high. These two falls divide the Rivière Nastapoka into three major segments: 1) a short estuary downstream from the first falls; 2) a second segment between the first and the second falls at $40-80 \mathrm{~m}$ asl; and 3) a third one upstream from the second falls at $>125 \mathrm{~m}$ asl.

\section{METHODS}

The landforms associated with raised coastlines were first mapped from interpretation of 1:10 000 and 1:20 000 aerial photographs. The marine limit was defined at numerous locations, mainly with the upper limit of the washing zone of ablation till on the tops and sides of rocky hills (Allard and Seguin, 1985); several glaciofluvial deltas are also at the same elevation. Downwards from this maximum level reached by the Tyrrell Sea at deglaciation time, other features indicative of past sea levels include raised boulder fields, many of them having wave-made boulder ridges, sandy beaches and the surface of perched deltas along river courses. Their elevation was measured at numerous places during field traverses. Natural sections of glacial, marine, deltaic, coastal and eolian sediments, exposed along gullies and rivers were described and logged (Fig. 2). Landform and sample elevations were determined with the help of a precision altimeter $(0.1 \mathrm{~m})$ using a provincial geodetic benchmark located near base camp as reference. Marine shells, wood fragments and organic paleosoils were collected for ${ }^{14} \mathrm{C}$ dating. Ages are reported here in

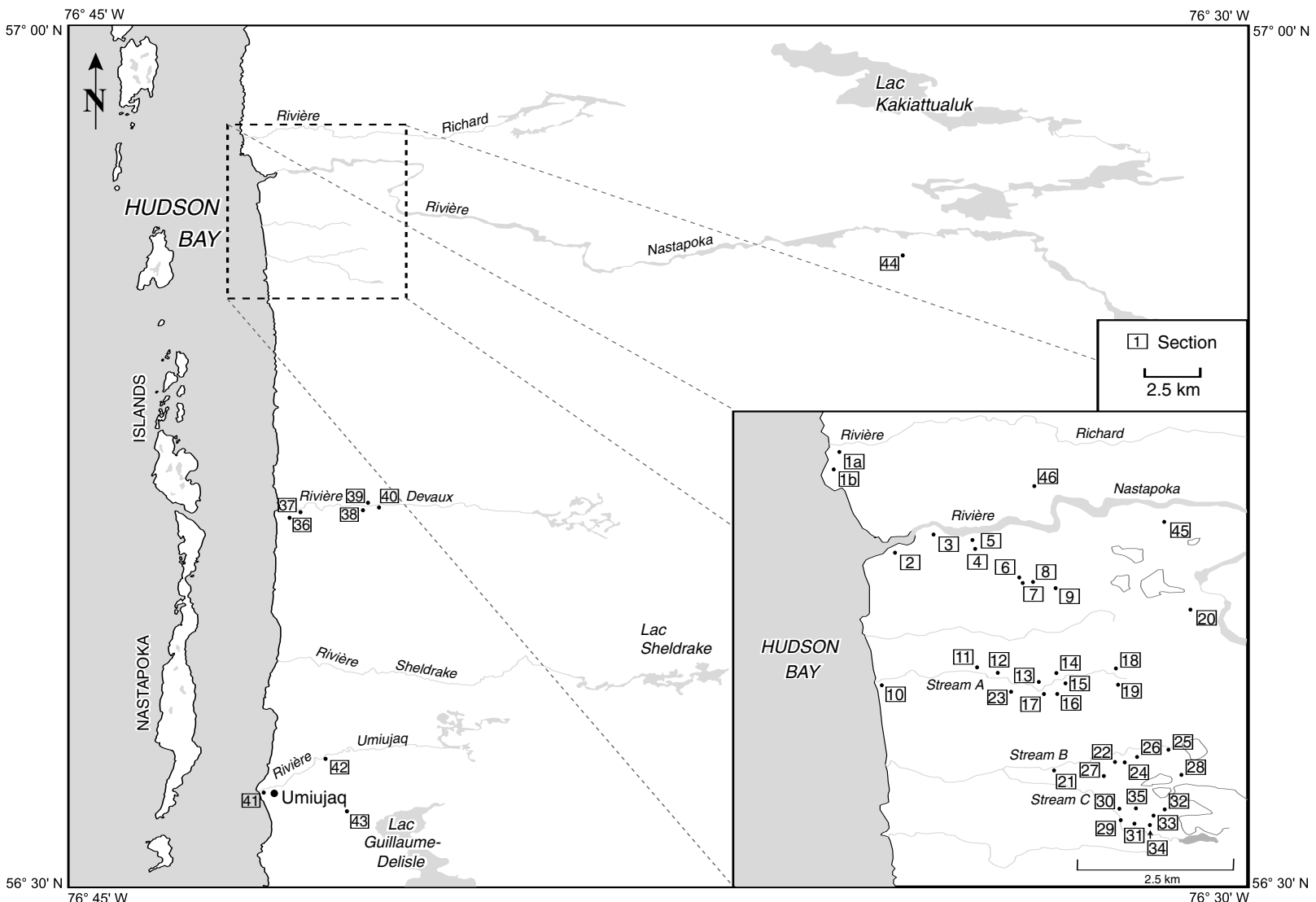

FIGURE 2. Location map of studied sections reported in text, Rivière

Carte de localisation des coupes étudiées et signalées dans le texte, Nastapoka area. dans la région de la rivière Nastapoka. 
conventional $\delta^{13} \mathrm{C}$ normalized ${ }^{14} \mathrm{C}$ and calibrated (cal.) years BP (Tabl. I; Fig. 3), in contrast to measured ${ }^{14} \mathrm{C}$ dates in Lajeunesse and Allard (2003). The dates were calibrated to calendar years by using the CALIB 4.3 program (Stuiver and Reimer, 1993). Calibration of shell dates was made using the $\Delta \mathrm{R}$ regional mean value of 310 years for the southeastern Hudson Bay area $\left({ }^{14} \mathrm{C}_{\text {meas. }}\right.$ - contemporaneous surface ocean ${ }^{14} \mathrm{C}$ age) (Barber et al., 1999). The intercept calendar year belonging to the range with the highest probability was retained as the calibrated date.

\section{GEOMORPHOLOGY}

\section{LATE-WISCONSINAN ICE FLOW}

Fluted till, striae, chattermarks, roches moutonnées and drumlins indicate a general westward $\left(270^{\circ}\right)$ flow that correspond to the regional ice flow reported in Parent et al. (1995). Flutings are located $>200 \mathrm{~m}$ asl and concentrated in the NE sector of the study area, although some are present north of Rivière du Nord (Fig. 4). They occur in sectors where the Tyrrell Sea waters did not invade the land, and above marine limit in areas where it did (Figs. 4,5). Bedrock outcrops exposing fresh and intact striae and associated erosion features are relatively rare since they were either eroded by periglacial coastal processes, affected by weathering or buried by Holocene sediments.

\section{ICE GROUNDING AND RECESSION POSITIONS}

Temporary stabilization phases of the ice margin are recorded by ice-contact landforms and sediments. These ice front stillstands occurred in marine and terrestrial environments and led to the construction of frontal, ablation and marine DeGeer moraines as well as ice-contact glaciomarine and glaciofluvial sediment bodies. The distribution of these features is almost exclusively restricted to sectors of relatively higher relief (Fig. 4). Frontal moraines are generally parallel to sub-parallel to the actual seashore and occur on some summits of the Nastapoka Hills, where they are clearly visible above marine limit. Frontal moraines also occur in the bottom of a wide erosion gully in a sandur-delta (S44) and near the downstream portion of an esker in the southeastern part of the area (Fig. 4). Grounding lines and morainal banks occur in a general north-south trend along the Nastapoka Hills and form a distinctive mark between sandy sediments westward and silty-clay sediments eastward.

Ablation till consists of sub-angular to sub-rounded boulders and gravel with a very thin and irregularly distributed blanket of poorly sorted sand. Boulder and gravel often lie directly on glacially moulded bedrock outcrops. Generally, the finer-grained material veneer locally smoothes the landscape by filling out small depressions in bedrock. This moraine was deposited in the ice marginal zone as the ice sheet was retreating. It occurs on most hilltops of the Nastapoka Hills and is almost completely eroded and reworked below the marine washing zone, which forms a clear distinctive mark of marine limit.

DeGeer moraines were observed at $180 \mathrm{~m}$ asl and $\sim 15 \mathrm{~km}$ north-east of the Rivière Nastapoka mouth. They are oriented parallel to the coast of Hudson Bay. As the marine limit in this sector is $\sim 200-210 \mathrm{~m}$ asl, these moraines were formed under $\sim 30 \mathrm{~m}$ of water. The mean spacing of $108 \mathrm{~m}$ between the ridges (Allard and Seguin, 1985), if taken as the yearly amount of retreat of the ice front, would suggest that it took $330 \mathrm{yr}$ for the ice front to retreat from the Nastapoka Hills to the inner limit of the marine transgression, some $35 \mathrm{~km}$ inland. This rate is less than the $150 \mathrm{~m}$ and $217 \mathrm{~m} / \mathrm{yr}$ measured further south in the James Bay area by Vincent (1977). This slower rate of retreat for the Nastapoka sector can be attributed to more pronounced local topographic variations, marked by deep valleys and hills higher than marine limit (Allard and Seguin, 1985).

Ice-contact glaciomarine sediment accumulations occur as submarine fans, sandur-deltas and deltas. These predominantly sandy bodies were constructed near the ice front during stabilization phases of the eastward retreating ice margin. These deposits form two drift belts: one along the Nastapoka Hills range and the other at the inner limit of marine inundation on the Hudsonian Plateau (Fig. 4). The first belt consists of ice-contact submarine fans that were deposited in a glaciomarine sedimentary environment during a period of high RSL (Lajeunesse and Allard, 2002) and a pause in ice recession (Lajeunesse and Allard, 2003). Some fans become coalescent and form aprons $\sim 5 \mathrm{~km}$ wide on the western slopes of the Nastapoka Hills. In many cases, sediments composing these fans are constricted in elongated bedrock basins and become coalescent downslope. The fans start upstream at $200-$ $220 \mathrm{~m}$ asl along the hill range (Lajeunesse and Allard, 2002) and slope down to below the present day sea level underneath Hudson Bay waters (Lavoie et al., 2002). Holocene gully erosion has exposed $>20 \mathrm{~m}$ of sand, silt, gravel beds containing abundant fossil shells. High energy jets of glacial meltwater carved large potholes of 1-1.5 m of diameter and 2-3 m deep in bedrock side-walls.

The second drift belt consists of ice-contact sandur-deltas located in the eastern part of the study area (Fig. 4). They are located at 195-205 $\mathrm{m}$ asl and are associated with both the marine limit and the inland postglacial marine overlap zone. Sediments composing sandur-deltas were deposited in both subaerial and submarine environments and contain topsets, foresets and bottomsets. The subaerial sandurs bear networks of braided channels. The length of the outwash extends on more than $12 \mathrm{~km}$ in some cases and are often pitted with kettles. Many sandur-deltas are located at the downstream end of long and sinuous eskers indicating a flow from east to west. A cross-section analysed in S44 located in the downstream sector of a sandur-delta exposes predominantly undisturbed fine sand and silt beds deposited by suspension-settling. Sediment deformation and minor loading structures occur, indicating rapid sedimentation. There are no fossil shells at this site, which can be attributed to high inputs of fresh water in a restrained embayment. The distance between two moraines of the DeGeer type buried under this section indicates a very slow glacial retreat of $15-20 \mathrm{~m} / \mathrm{yr}$, i.e. a near stillstand. This second drift belt either marks a pause or a drastic slowdown of glacial retreat as the glacier front became separated from the effect of sea waters. Mass wasting became regulated only by melt without any influence of calving. 
TABLE I

Radiocarbon and calibrated ages, with $68.3 \%(1 \sigma)$ confidence limits, from fossil shells, driftwood, terrestrial wood, peat and paleosoils

\begin{tabular}{|c|c|c|c|c|c|c|c|c|}
\hline $\begin{array}{l}\text { Lab. } \\
\text { No. }^{\text {a }}\end{array}$ & Location & $\begin{array}{l}\text { Elev. } \\
(\mathrm{m})\end{array}$ & $\begin{array}{l}\mathrm{RSL} \\
(\mathrm{m})\end{array}$ & $\begin{array}{c}\text { Age } \\
(\mathrm{yr} \mathrm{BP})^{\mathrm{b}}\end{array}$ & Cal. yr BP & Species dated & Unit & Ref. $^{d}$ \\
\hline UL-2346 & $57^{\circ} 00^{\prime} 18^{\prime \prime} \mathrm{N} 76^{\circ} 30^{\prime} 28^{\prime \prime} \mathrm{W}$ & 4 & $>26$ & $8430 \pm 120$ & $8770(8580) 8420$ & Macoma balthica & De1a & 1 \\
\hline UL-1932 & $56^{\circ} 51^{\prime} 04^{\prime \prime} \mathrm{N} 76^{\circ} 27^{\prime} 00^{\prime \prime} \mathrm{W}$ & 145 & $>155$ & $8140 \pm 120$ & $8580(8300) 8160$ & $\begin{array}{c}\text { Mya truncata } \\
\text { Hiatella arctica } \\
\text { Macoma balthica }\end{array}$ & De1a & 1 \\
\hline UL-1951 & $56^{\circ} 52^{\prime} 40^{\prime \prime} \mathrm{N} 76^{\circ} 32^{\prime} 15^{\prime \prime} \mathrm{W}$ & 108 & $>120$ & $7770 \pm 130$ & $8020(7920) 7780$ & Hiatella arctica & $\mathrm{Em}$ & 1 \\
\hline TO-7411 & $56^{\circ} 51^{\prime} 04^{\prime \prime} \mathrm{N} 76^{\circ} 27^{\prime} 00^{\prime \prime} \mathrm{W}$ & 154 & $>155$ & $7700 \pm 70$ & 7920 (7840) 7760 & $\begin{array}{c}\text { Mya truncata } \\
\text { Macoma calcarea }\end{array}$ & De1a & 1 \\
\hline UL-1740 & $56^{\circ} 55^{\prime} 00^{\prime \prime} \mathrm{N} 76^{\circ} 29^{\prime} 09^{\prime \prime} \mathrm{W}$ & 115 & $>115$ & $7620 \pm 90$ & 7850 (7750) 7670 & Hiatella arctica & De2 & 1 \\
\hline UL-1728 & $56^{\circ} 54^{\prime} 04^{\prime \prime} \mathrm{N} 76^{\circ} 29^{\prime} 45^{\prime \prime} \mathrm{W}$ & 110 & $>110$ & $7570 \pm 80$ & 7790 (7700) 7640 & Hiatella arctica & De1a & 1 \\
\hline UL-1729 & $56^{\circ} 52^{\prime} 56^{\prime \prime} \mathrm{N} 76^{\circ} 32^{\prime} 15^{\prime \prime} \mathrm{W}$ & 93 & $>120$ & $7440 \pm 80$ & $7660(7590) 7550$ & Hiatella arctica & De1a & 1 \\
\hline CAMS-37437 & $56^{\circ} 52^{\prime} 55^{\prime \prime} \mathrm{N} 76^{\circ} 32^{\prime} 45^{\prime \prime} \mathrm{W}$ & 5 & $>7$ & $7370 \pm 60$ & $8280(8180) 8060$ & Driftwood & Em & 4 \\
\hline UL-1722 & $56^{\circ} 52^{\prime} 56^{\prime \prime} \mathrm{N} 76^{\circ} 32^{\prime} 15^{\prime \prime} \mathrm{W}$ & 95 & $>120$ & $7330 \pm 80$ & $7570(7500) 7430$ & Hiatella arctica & De1a & 1 \\
\hline UL-1937 & $56^{\circ} 52^{\prime} 00^{\prime \prime} \mathrm{N} 76^{\circ} 26^{\prime} 45^{\prime \prime} \mathrm{W}$ & 132 & $>146$ & $7320 \pm 80$ & $7570(7500) 7420$ & $\begin{array}{l}\text { Mytilus edulis } \\
\text { Hiatella arctica }\end{array}$ & De1a & 1 \\
\hline TO-7412 & $56^{\circ} 55^{\prime} 00^{\prime \prime} \mathrm{N} 76^{\circ} 29^{\prime} 15^{\prime \prime} \mathrm{W}$ & 133 & $>136$ & $7150 \pm 90$ & 7430 (7360) 7260 & $\begin{array}{c}\text { Hiatella arctica } \\
\text { Buccinum undatum } \\
\text { Balanus sp. }\end{array}$ & De2 & 1 \\
\hline UL-1718 & $56^{\circ} 54^{\prime} 04^{\prime \prime} \mathrm{N} 76^{\circ} 29^{\prime} 45^{\prime \prime} \mathrm{W}$ & 112 & $>112$ & $7150 \pm 80$ & $7420(7360) 7270$ & $\begin{array}{l}\text { Hiatella arctica } \\
\text { Mytilus edulis }\end{array}$ & De1a & 1 \\
\hline UQ-547 & $56^{\circ} 54^{\prime} 00^{\prime \prime} \mathrm{N} 76^{\circ} 26^{\prime} 30^{\prime \prime} \mathrm{W}$ & 180 & n.d. & $7110 \pm 100$ & $7110(7310) 7230$ & $\begin{array}{c}\text { Hiatella arctica } \\
\text { Mytilus edulis }\end{array}$ & De2 & 2 \\
\hline UL-1709 & $56^{\circ} 52^{\prime} 55^{\prime \prime} \mathrm{N} 76^{\circ} 29^{\prime} 45^{\prime \prime} \mathrm{W}$ & 101 & $>114$ & $7080 \pm 90$ & 7390 (7290) 7220 & Mytilus edulis & De1a & 1 \\
\hline UL-1717 & $56^{\circ} 52^{\prime} 55^{\prime \prime} \mathrm{N} 76^{\circ} 29^{\prime} 45^{\prime \prime} \mathrm{W}$ & 107 & $>110$ & $7070 \pm 80$ & 7370 (7270) 7220 & Hiatella arctica & De1a & 1 \\
\hline UL-1953 & $56^{\circ} 52^{\prime} 00^{\prime \prime} \mathrm{N} 76^{\circ} 26^{\prime} 15^{\prime \prime} \mathrm{W}$ & 139 & $>139$ & $7050 \pm 80$ & $7330(7260) 7200$ & Mytilus edulis & De1a & 1 \\
\hline UQ-545 & $56^{\circ} 53^{\prime} 30^{\prime \prime} \mathrm{N} 76^{\circ} 25^{\prime} 30^{\prime \prime} \mathrm{W}$ & 120 & n.d. & $7010 \pm 100$ & $7310(7240) 7150$ & $\begin{array}{c}\text { Hiatella arctica } \\
\text { Mytilus edulis }\end{array}$ & De2 & 2 \\
\hline UL-1711 & $56^{\circ} 52^{\prime} 55^{\prime \prime} \mathrm{N} 76^{\circ} 29^{\prime} 45^{\prime \prime} \mathrm{W}$ & 109 & $>110$ & $6980 \pm 70$ & $7260(7210) 7150$ & $\begin{array}{c}\text { Mytilus edulis } \\
\text { Hiatella arctica } \\
\text { Chlamys islandicus }\end{array}$ & De1a & 1 \\
\hline UL-1928 & $56^{\circ} 51^{\prime} 48^{\prime \prime} \mathrm{N} 76^{\circ} 26^{\prime} 15^{\prime \prime} \mathrm{W}$ & 146 & $>148$ & $6950 \pm 80$ & $7250(7180) 7080$ & $\begin{array}{l}\text { Hiatella arctica } \\
\text { Mytilus edulis }\end{array}$ & De1a & 1 \\
\hline UL-1918 & $56^{\circ} 52^{\prime} 55^{\prime \prime} \mathrm{N} 76^{\circ} 29^{\prime} 45^{\prime \prime} \mathrm{W}$ & 104 & $>104$ & $6920 \pm 110$ & $7250(7150) 6990$ & $\begin{array}{l}\text { Mytilus edulis } \\
\text { Hiatella arctica }\end{array}$ & De1a & 1 \\
\hline Beta-121551 & $56^{\circ} 48^{\prime} 12^{\prime \prime} \mathrm{N} 76^{\circ} 26^{\prime} 15^{\prime \prime} \mathrm{W}$ & 146 & $>154$ & $6890 \pm 70$ & $7200(7130) 7000$ & $\begin{array}{l}\text { Hiatella arctica } \\
\text { Mytilus edulis }\end{array}$ & De1a & 1 \\
\hline TO-7410 & $56^{\circ} 52^{\prime} 55^{\prime \prime} \mathrm{N} 76^{\circ} 29^{\prime} 45^{\prime \prime} \mathrm{W}$ & 108 & $>114$ & $6870 \pm 60$ & 7160 (7080) 6990 & $\begin{array}{l}\text { Hiatella arctica } \\
\text { Mytilus edulis }\end{array}$ & De1a & 1 \\
\hline Beta-121552 & $56^{\circ} 32^{\prime} 30^{\prime \prime} \mathrm{N} 76^{\circ} 27^{\prime} 30^{\prime \prime} \mathrm{W}$ & 100 & $>100$ & $6840 \pm 70$ & 7150 (7020) 6940 & $\begin{array}{l}\text { Mytilus edulis } \\
\text { Hiatella arctica }\end{array}$ & De1b & 1 \\
\hline UL-1555 & $56^{\circ} 54^{\prime} 15^{\prime \prime} \mathrm{N} 76^{\circ} 29^{\prime} 50^{\prime \prime} \mathrm{W}$ & 85 & $>85$ & $6660 \pm 110$ & $6940(6290) 6670$ & Undiff. shells & De1a & \\
\hline UL-1952 & $56^{\circ} 48^{\prime} 09^{\prime \prime} \mathrm{N} 76^{\circ} 28^{\prime} 10^{\prime \prime} \mathrm{W}$ & 84 & $>84$ & $6600 \pm 80$ & $6840(6730) 6650$ & $\begin{array}{l}\text { Hiatella arctica } \\
\text { Mytilus edulis }\end{array}$ & De1 & 1 \\
\hline UQ-540 & $56^{\circ} 53^{\prime} 30^{\prime \prime} \mathrm{N} 76^{\circ} 29^{\prime} 50^{\prime \prime} \mathrm{W}$ & 118 & $>118$ & $6530 \pm 80$ & $6780(6660) 6530$ & $\begin{array}{l}\text { Hiatella arctica } \\
\text { Mytilus edulis }\end{array}$ & n.d. & 4 \\
\hline UL-1540 & $56^{\circ} 52^{\prime} 45^{\prime \prime} \mathrm{N} 76^{\circ} 29^{\prime} 50^{\prime \prime} \mathrm{W}$ & 97 & $>97$ & $6530 \pm 110$ & $6780(6660) 6530$ & Hiatella arctica & De1a & 4 \\
\hline UL-1710 & $56^{\circ} 52^{\prime} 55^{\prime \prime} \mathrm{N} 76^{\circ} 29^{\prime} 45^{\prime \prime} \mathrm{W}$ & 110 & $>110$ & $6450 \pm 110$ & $6700(6570) 6690$ & $\begin{array}{l}\text { Mytilus edulis } \\
\text { Hiatella arctica }\end{array}$ & De1a & 1 \\
\hline TO-7409 & $56^{\circ} 52^{\prime} 55^{\prime \prime} \mathrm{N} 76^{\circ} 29^{\prime} 45^{\prime \prime} \mathrm{W}$ & 109 & $>114$ & $6420 \pm 60$ & $6620(6530) 6450$ & Mya truncata & De1a & 1 \\
\hline I-13406 & $56^{\circ} 52^{\prime} 30^{\prime \prime} \mathrm{N} 76^{\circ} 23^{\prime} 00^{\prime \prime} \mathrm{W}$ & 180 & $<180$ & $6080 \pm 80$ & 7150 (6910) 6760 & Peat & n.d. & 2 \\
\hline
\end{tabular}


TABLE I (continued)

Radiocarbon and calibrated ages, with $68.3 \%(1 \sigma)$ confidence limits, from fossil shells, driftwood, terrestrial wood, peat and paleosoils

\begin{tabular}{|c|c|c|c|c|c|c|c|c|}
\hline $\begin{array}{l}\text { Lab. } \\
\text { No. }^{a}\end{array}$ & Location & $\begin{array}{l}\text { Elev. } \\
(\mathrm{m})\end{array}$ & $\begin{array}{l}\text { RSL } \\
(\mathrm{m})\end{array}$ & $\begin{array}{c}\text { Age } \\
(y r \text { BP })^{\mathrm{b}}\end{array}$ & Cal. yr BPc & Species dated & Unit & Ref. $^{d c}$ \\
\hline $\mathrm{I}-13130$ & $56^{\circ} 52^{\prime} 30^{\prime \prime} \mathrm{N} 76^{\circ} 23^{\prime} 00^{\prime \prime} \mathrm{W}$ & 170 & $<170$ & $5840 \pm 150$ & $6850(6660) 6450$ & Peat & n.d. & 4 \\
\hline I-13131 & $56^{\circ} 52^{\prime} 30^{\prime \prime} \mathrm{N} 76^{\circ} 23^{\prime} 00^{\prime \prime} \mathrm{W}$ & 170 & $<170$ & $5810 \pm 120$ & $6750(6640) 6450$ & Wood & n.d. & 4 \\
\hline UL-1556 & $56^{\circ} 54^{\prime} 27^{\prime \prime} \mathrm{N} 76^{\circ} 31^{\prime} 56^{\prime \prime} \mathrm{W}$ & 51 & $>100$ & $5730 \pm 100$ & $5900(5760) 5660$ & Driftwood & $\mathrm{Pa} 3$ & 4 \\
\hline CAMS-37434 & $56^{\circ} 52^{\prime} 55^{\prime \prime} \mathrm{N} 76^{\circ} 32^{\prime} 45^{\prime \prime} \mathrm{W}$ & 5 & $>7$ & $4780 \pm 60$ & $4780(4630) 4540$ & Chlamys islandicus & $\mathrm{Em}$ & 4 \\
\hline UL-1904 & $56^{\circ} 52^{\prime} 55^{\prime \prime} \mathrm{N} 76^{\circ} 29^{\prime} 45^{\prime \prime} \mathrm{W}$ & 98 & $<98$ & $4630 \pm 100$ & $5570(5420) 5090$ & Paleosoil & $\mathrm{Em}$ & 4 \\
\hline UL-1919 & $56^{\circ} 47^{\prime} 45^{\prime \prime} \mathrm{N} 76^{\circ} 31^{\prime} 40^{\prime \prime} \mathrm{W}$ & 13 & $>25$ & $4540 \pm 70$ & 4410 (4340) 4230 & $\begin{array}{c}\text { Mytilus edulis } \\
\text { Chlamys islandicus } \\
\text { Macoma balthica } \\
\text { Hiatella arctica } \\
\text { Crenella gladulosa } \\
\text { Balanus sp. } \\
\text { Mya truncata }\end{array}$ & $\mathrm{Pa} 3$ & 4 \\
\hline UL-1917 & $56^{\circ} 47^{\prime} 45^{\prime \prime} \mathrm{N} 76^{\circ} 31^{\prime} 40^{\prime \prime} \mathrm{W}$ & 15 & $>25$ & $4220 \pm 70$ & 3970 (3870) 3810 & $\begin{array}{c}\text { Hiatella arctica } \\
\text { Macoma Balthica } \\
\text { Chlamys islandicus } \\
\text { Balanus sp. } \\
\text { Buccinum undatum }\end{array}$ & Pa3 & 4 \\
\hline UL-1878 & $56^{\circ} 55^{\prime} 00^{\prime \prime} \mathrm{N} 76^{\circ} 29^{\prime} 15^{\prime \prime} \mathrm{W}$ & 136 & $<136$ & $4190 \pm 100$ & $4850(4750) 4540$ & Paleosoil & $\mathrm{Em}$ & 4 \\
\hline UL-1546 & $56^{\circ} 55^{\prime} 45^{\prime \prime} \mathrm{N} 76^{\circ} 33^{\prime} 45^{\prime \prime} \mathrm{W}$ & 12 & $>13$ & $3960 \pm 90$ & 3660 (3550) 3440 & $\begin{array}{l}\text { Clinocardium ciliatum } \\
\text { Buccinum undatum }\end{array}$ & Em & 4 \\
\hline UL-1371 & $56^{\circ} 30^{\prime} 00^{\prime \prime} \mathrm{N} 76^{\circ} 26^{\prime} 15^{\prime \prime} \mathrm{W}$ & n.d. & n.d. & $3540 \pm 70$ & $3150(3040) 2940$ & Hiatella arctica & Pa1 & 4 \\
\hline UL-1903 & $56^{\circ} 52^{\prime} 00^{\prime \prime} \mathrm{N} 76^{\circ} 26^{\prime} 15^{\prime \prime} \mathrm{W}$ & 150 & $<150$ & $2040 \pm 60$ & 2100 (1960) 1900 & Paleosoil & Em & 4 \\
\hline UL-1949 & $56^{\circ} 54^{\prime} 04^{\prime \prime} \mathrm{N} 76^{\circ} 28^{\prime} 30^{\prime \prime} \mathrm{W}$ & 133 & $<133$ & $840 \pm 100$ & $910(730) 670$ & Paleosoil & $\mathrm{Em}$ & 4 \\
\hline UL-1536 & $56^{\circ} 55^{\prime} 20^{\prime \prime} \mathrm{N} 76^{\circ} 34^{\prime} 38^{\prime \prime} \mathrm{W}$ & 4 & $>\quad 4.5$ & $460 \pm 80$ & $541(510) 470$ & Driftwood & Em & 4 \\
\hline UL-1528 & $56^{\circ} 55^{\prime} 20^{\prime \prime} \mathrm{N} 76^{\circ} 34^{\prime} 38^{\prime \prime} \mathrm{W}$ & 4 & $>4.5$ & $440 \pm 70$ & $530(510) 460$ & Driftwood & $\mathrm{Em}$ & 4 \\
\hline UL-1858 & $56^{\circ} 55^{\prime} 20^{\prime \prime} \mathrm{N} 76^{\circ} 34^{\prime} 38^{\prime \prime} \mathrm{W}$ & 12 & $<12$ & $280 \pm 90$ & $470(310) 150$ & Paleosoil & Em & 4 \\
\hline UL-1946 & $56^{\circ} 48^{\prime} 12^{\prime \prime} \mathrm{N} 76^{\circ} 26^{\prime} 15^{\prime \prime} \mathrm{W}$ & 12 & $<12$ & Modern & Modern & Paleosoil & $\mathrm{Em}$ & 4 \\
\hline UL-1859 & $56^{\circ} 55^{\prime} 20^{\prime \prime} \mathrm{N} 76^{\circ} 34^{\prime} 38^{\prime \prime} \mathrm{W}$ & 13 & $<13$ & Modern & Modern & Paleosoil & $\mathrm{Em}$ & 4 \\
\hline
\end{tabular}

a Laboratory designation: UQ = Université du Québec à Montréal, UL = Université Laval, CAMS = Lawrence Livermore National Laboratory, University of California, TO = Isotrace Laboratory, University of Toronto, Beta = Beta Analytic, I = Teledyne Isotopes, Westwood, New Jersey.

${ }^{b}{ }^{14} \mathrm{C}$ dates are conventional, normalized to $\delta^{13} \mathrm{C}=-25 \%$.

${ }^{c}$ Calibrated ages, see text for calibration procedure.

d 1: Lajeunesse and Allard (2003), 2: Allard and Seguin (1985), 3: Allard and Seguin (1987), 4: this paper.

\section{MARINE LIMIT AND UPLIFTED SHORELINES}

Marine limit is at $248 \mathrm{~m}$ asl near the Rivière Nastapoka mouth, 220-227 $\mathrm{m}$ asl north of Rivière Nastapoka and $230 \mathrm{~m}$ asl at Rivière Devaux (Fig. 5). East of the Nastapoka Hills, the plane of the marine limit slopes down to $204 \mathrm{~m}$ asl at the maximum inland reach of the Tyrrell Sea where it forms an irregular paleo-coastline pattern (Allard and Seguin, 1985). Marine invasion went as far as $56 \mathrm{~km}$ inland from the present day coastline at Lac Kakiattualuk, 48 km along Rivière Nastapoka, $23 \mathrm{~km}$ along Rivière Devaux and $67 \mathrm{~km}$ in the southern portion of the study area in the depression of Lac Guillaume-Delisle.

Postglacial emergence is recorded by boulder ridges and pavements, raised beaches, perched deltas and terraces as well as coastal gelifraction of bedrock. Boulder ridges and pavements occur at any elevation below marine limit. Between 248 and $200 \mathrm{~m}$ asl, where bedrock has absolutely no cover of fines, they represent, together with gelifracted outcrops, the main geomorphic record of land emergence. Boulder ridges generally form series of linear or lightly curved ridges on sidewalls of small valleys. Boulder pavements occur on flat or gently sloping surfaces. Coastal periglacial erosion features (i.e. landforms made by frost-riving and ice-push processes on the shore) can be found up to the very marine limit. Below this limit, the smooth glacially moulded surface of bedrock was intensely disrupted by frost action in a wet coastal environment into angular clasts which were then carried away by drift ice and storm waves (Allard and Tremblay, 1983b). A large number of these clasts are present in boulder barricades and pavements 


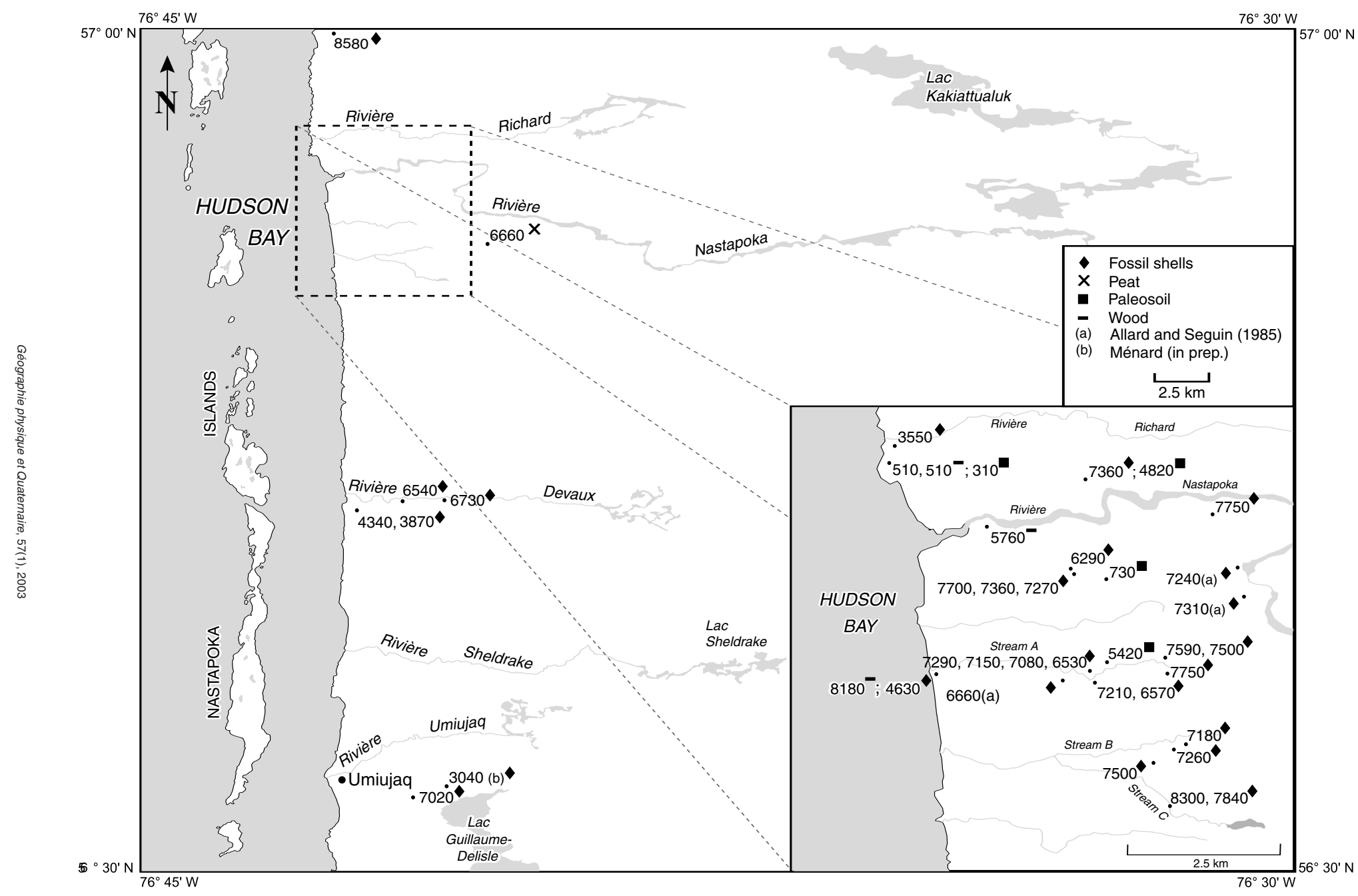




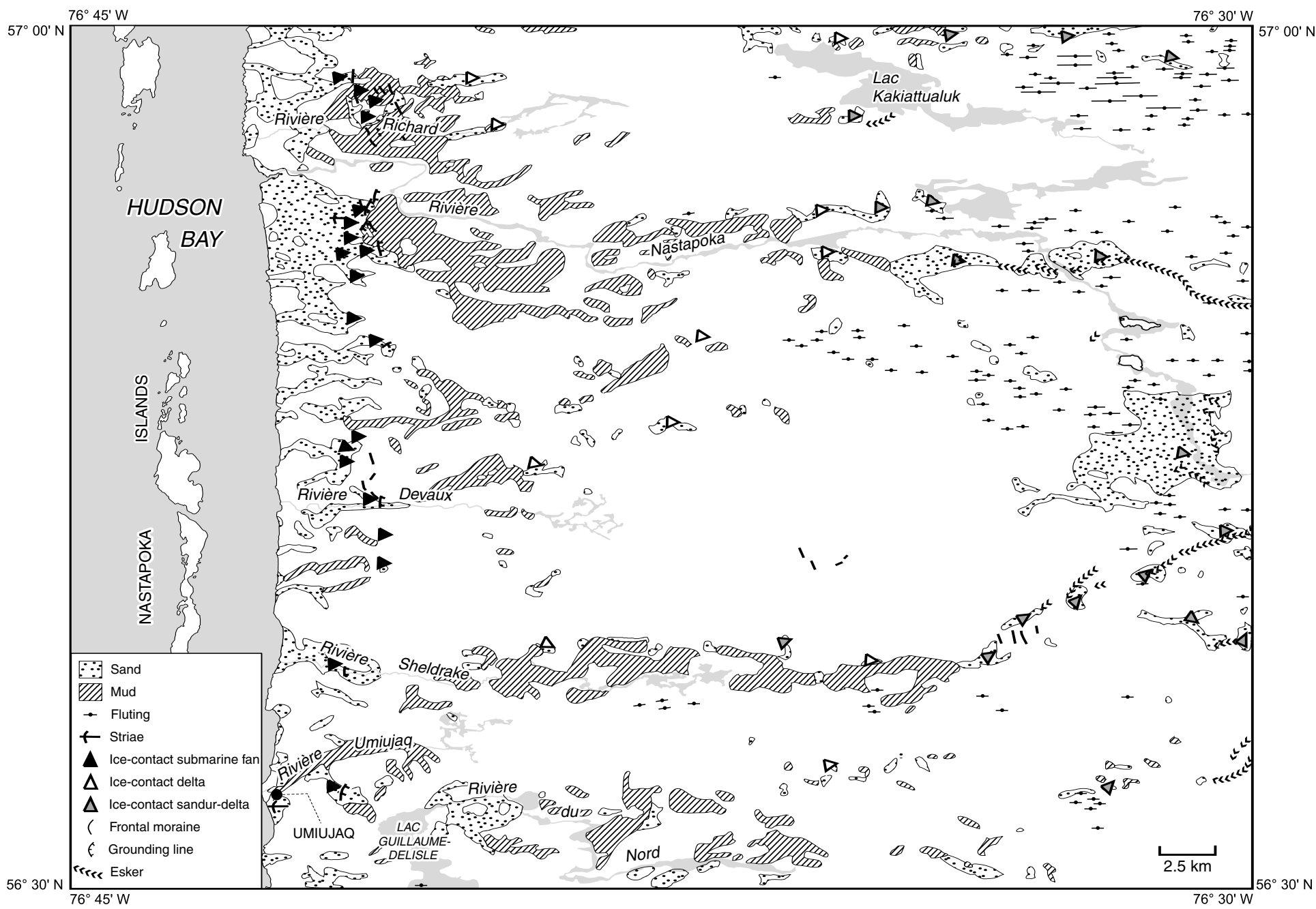




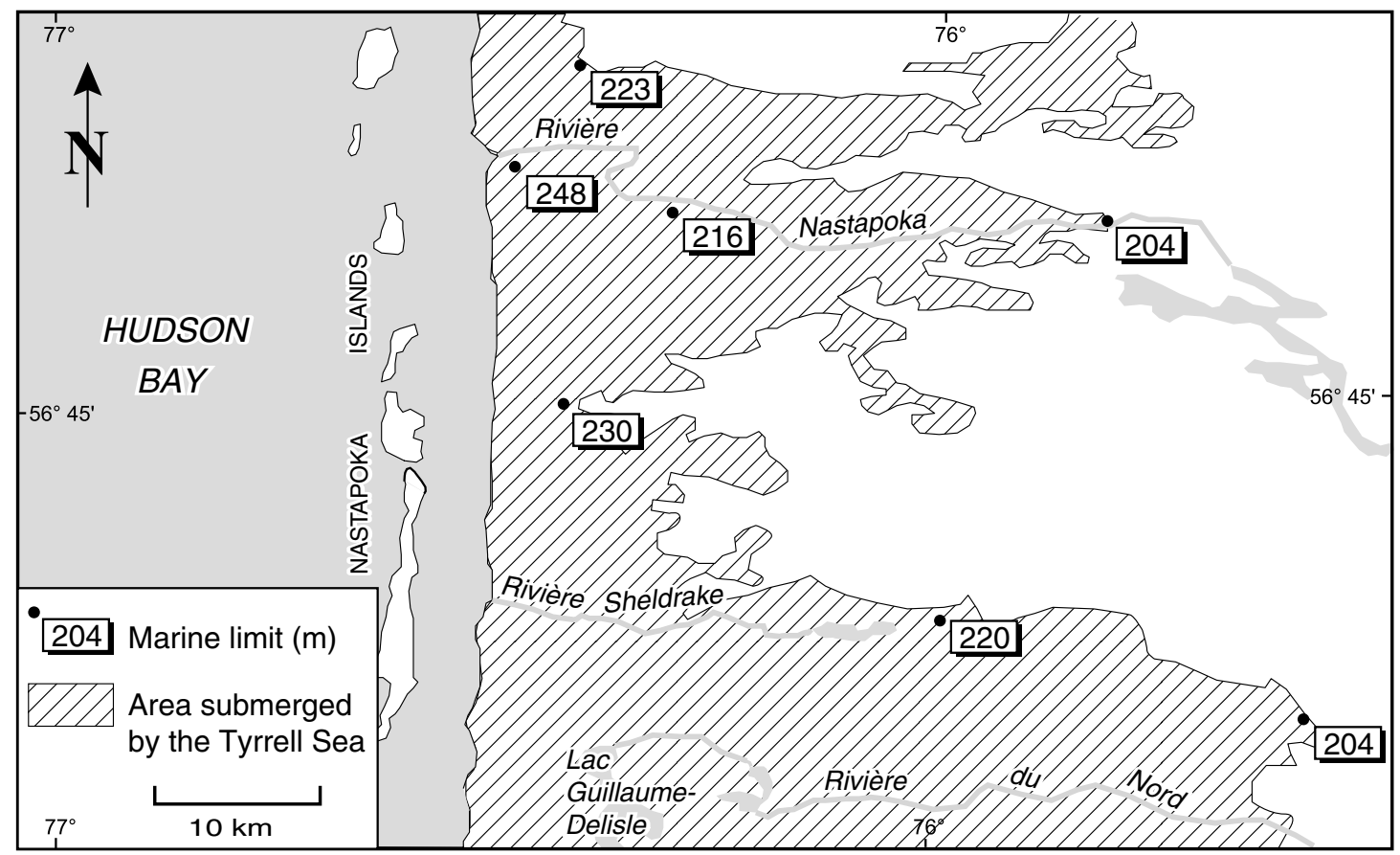

FIGURE 5. Map of the Tyrrell Sea extent in the study area, eastern Hudson Bay (after Allard and Seguin [1985]; modified by Lévesque et al. [1988] and Lavoie [2000]).

and are mixed with subrounded allochtonous clasts derived from the washing out of glacial drift (Dionne, 1978). Coastal periglacial processes are still active along the present day shoreline. Series of beach ridges occur below $200 \mathrm{~m}$ asl, where they often form almost regular staircases in sand deposits. Vertical spacing between these ridges ranges from 2 to $7 \mathrm{~m}$, as in the Lac Guillaume-Delisle region, just south of the study area (Hillaire-Marcel, 1976). These ridges are common features on the surface of the emerged deposits of the western slopes of the Nastapoka Hills. At least 60 beach crests occur between 0 and $100 \mathrm{~m}$ asl on a distance of $2.5 \mathrm{~km}$ near the Rivière Nastapoka mouth.

\section{SEDIMENTOLOGY AND STRATIGRAPHY}

\section{RAISED LATE QUATERNARY DEPOSITS}

With the exception of till and moraines, Late Quaternary sediments of the study area record depositional and reworking processes that took place during the initial phase of marine transgression and the subsequent falling RSL. Sediments exposed in sections were deposited in ice-proximal (De1) and ice-distal (De2) glaciomarine, paraglacial $(\mathrm{Pa})$ and emergence (Em) depositional environments (Tabl. II; Fig. 6). De1 deposits are associated with the two stillstands of the ice front (Nastapoka Hills glaciomarine fan complex and sandur-delta
Carte de l'extension de la Mer de Tyrrell dans la région d'étude (d'après Allard et Seguin, 1985; modifiée par Lévesque et al., 1988, et Lavoie, 2000).

belt), De2 sediments are associated with the retreat between these two ice front positions. Pa sediments are associated with fluvial erosion and sedimentation as rivers made new courses by down-cutting and re-depositing existing sediments. A coarsening upward sequence representing the Holocene falling RSL is observed in most sections.

\section{Deglacial deposits: ice-proximal sediments (De1)}

Ice-proximal sediments (De1) are dominated by sand (Fig. 6). These deposits are concentrated on the coastal slope between 80 and $220 \mathrm{~m}$ asl, where they form thick and extensive submarine fans (Lajeunesse and Allard, 2002), and in the eastern part of the study area between $\sim 185-220 \mathrm{~m}$ asl, where they form deltas and sandur-deltas. The largest deposits occur near the Rivière Nastapoka mouth and along the river in the eastern end of the studied sector. Smaller and restricted accumulations of De1 sediments occur in the inner basin and are associated with ice-contact deltas. In the Nastapoka Hills, De1 deposits extend from 0 to $4 \mathrm{~km}$ downstream from the former ice front. Exposures of De1 are up to $23 \mathrm{~m}$ high in sections S32, S39-S40 and S42. De1 are exposed in S1a, S6-S9, S12-S19, S21-S35, S38-S40 and S42-S44 (Fig. 2). De1 is divided in members De1a and De1b, which correspond respectively to FA I and FA II in Lajeunesse and Allard (2002). De1a sediments consist of interbedded and 
TABLE II

Depositional processes and environments associated to the emerged marine deposits in the Rivière Nastapoka area

\begin{tabular}{|c|c|c|c|c|c|c|}
\hline Unit & Facies association & Characteristics & Depositional processes & Stratigraphy & $\begin{array}{c}\text { Age } \\
\text { (cal BP) }\end{array}$ & $\begin{array}{l}\text { Depositional } \\
\text { environment }\end{array}$ \\
\hline $\begin{array}{l}\text { Ice-proximal } \\
\text { glaciomarine } \\
\text { (De1a) }\end{array}$ & $\begin{array}{l}\text { Interbedded and } \\
\text { interlaminated } \\
\text { sand, silt and mud }\end{array}$ & $\begin{array}{l}\text { Deformation and dewatering } \\
\text { structures; normal grading; } \\
\text { normal faults; ripples; cross-beds; } \\
\text { massive sand beds with outsized } \\
\text { and mud clasts; semi- } \\
\text { consolidated beds; fossiliferous }\end{array}$ & $\begin{array}{l}\text { Submarine debris flows; high } \\
\text { and low density turbidity } \\
\text { currents; suspension from } \\
\text { turbid plumes (cyclopsams); } \\
\text { channel fill; traction-plus- } \\
\text { fallout; ice-rafting }\end{array}$ & $\begin{array}{l}\text { Lies on bedrock, } \\
\text { recovered by emergence } \\
\text { facies with erosive } \\
\text { contacts; locally lies } \\
\text { above ice-distal mud } \\
\text { (contact not observed) }\end{array}$ & $\begin{array}{c}8300 \pm 120 \\
\text { to } \\
6530 \pm 60\end{array}$ & $\begin{array}{l}\text { Ice-contact submarine } \\
\text { fans (activity of slope } \\
\text { failures remained active } \\
\text { until } ~ 6.1 \text { cal. ka BP); } \\
\text { Ice-contact deltas and } \\
\text { sandur-deltas }\end{array}$ \\
\hline $\begin{array}{l}\text { Ice-proximal } \\
\text { glaciomarine } \\
\text { (De1b) }\end{array}$ & Massive diamict & $\begin{array}{l}\text { Boulders within sand matrix; } \\
\text { scoured and erosive lower } \\
\text { contacts; unfossiliferous; semi- } \\
\text { consolidated beds }\end{array}$ & $\begin{array}{l}\text { Submarine debris flow; ice } \\
\text { rafting }\end{array}$ & $\begin{array}{l}\text { Interfingers with ice- } \\
\text { proximal sand, silt and } \\
\text { mud De1a with an } \\
\text { erosive lower contact }\end{array}$ & - & $\begin{array}{l}\text { Ice-contact submarine } \\
\text { fans }\end{array}$ \\
\hline $\begin{array}{l}\text { Ice-distal } \\
\text { glaciomarine } \\
\text { (De2a) }\end{array}$ & $\begin{array}{l}\text { Massive to } \\
\text { laminated mud and } \\
\text { sand }\end{array}$ & $\begin{array}{l}\text { Massive and laminated; } \\
\text { deformation structures; rare } \\
\text { outsized clasts; sparsely } \\
\text { fossiliferous; }\end{array}$ & $\begin{array}{l}\text { Suspension from turbid } \\
\text { plumes; cyclopels }\end{array}$ & $\begin{array}{l}\text { Lies on bedrock and } \\
\text { interfinger with ice-distal } \\
\text { blue-grey laminated mud } \\
\text { De2b }\end{array}$ & $\begin{array}{l}7750 \pm 130 \\
\text { to } \\
7240 \pm 80\end{array}$ & $\begin{array}{l}\text { Ice-distal glacial turbid } \\
\text { plumes; rain-out }\end{array}$ \\
\hline $\begin{array}{l}\text { Ice-distal } \\
\text { glaciomarine } \\
\text { (De2b) }\end{array}$ & $\begin{array}{l}\text { Massive to } \\
\text { laminated mud }\end{array}$ & $\begin{array}{l}\text { Blue-grey colour; black mono- } \\
\text { sulphide layers; sulphur odour; } \\
\text { varves }\end{array}$ & $\begin{array}{l}\text { Suspension from turbid } \\
\text { plumes; cyclopels; annual } \\
\text { layers }\end{array}$ & $\begin{array}{l}\text { Lies on bedrock and } \\
\text { interfinger with ice-distal } \\
\text { mud De2 }\end{array}$ & & $\begin{array}{l}\text { Ice-distal glacial turbid } \\
\text { plumes; rain-out }\end{array}$ \\
\hline $\begin{array}{l}\text { Paraglacial } \\
(\mathrm{Pa} 1)\end{array}$ & $\begin{array}{l}\text { Interbedded and } \\
\text { interlaminated mud } \\
\text { and sand }\end{array}$ & $\begin{array}{l}\text { Dark-grey or blue-grey colour; } \\
\text { organic layers enclosing driftwood }\end{array}$ & Suspension & $\begin{array}{l}\text { Lies above ice-distal mud } \\
\text { (contact not observed) }\end{array}$ & $5760 \pm 100$ & $\begin{array}{l}\text { Paraglacial prodeltaic } \\
\text { (distal) }\end{array}$ \\
\hline $\begin{array}{l}\text { Paraglacial } \\
(\mathrm{Pa} 2)\end{array}$ & $\begin{array}{l}\text { Interbedded sand, } \\
\text { silt and/or mud }\end{array}$ & $\begin{array}{l}\text { Rhythmites; ripples; plane and } \\
\text { wavy laminations; asymmetric } \\
\text { wavy laminations }\end{array}$ & $\begin{array}{l}\text { Suspension; traction-plus- } \\
\text { fallout; }\end{array}$ & $\begin{array}{l}\text { Lies above } \mathrm{Pa} 1 \text { with a } \\
\text { gradational contacts }\end{array}$ & - & $\begin{array}{l}\text { Paraglacial prodeltaic } \\
\text { (distal foresets) }\end{array}$ \\
\hline $\begin{array}{l}\text { Paraglacial } \\
(\mathrm{Pa} 3)\end{array}$ & $\begin{array}{l}\text { Laminated and } \\
\text { massive sand }\end{array}$ & $\begin{array}{l}\text { Subplanar to inclined massive } \\
\text { beds and laminations; gravel } \\
\text { beds; flaser beds; large-scale } \\
\text { cross-bedding }\end{array}$ & Traction (bedload); channel fill & $\begin{array}{l}\text { Lies above } \mathrm{Pa} 2 \text { with a } \\
\text { gradational contact }\end{array}$ & $\begin{aligned} 4330 & \pm 70 \\
\text { to } & \\
3870 & \pm 70\end{aligned}$ & $\begin{array}{l}\text { Paraglacial deltaic } \\
\text { (foresets) }\end{array}$ \\
\hline $\begin{array}{l}\text { Emergence } \\
(\mathrm{Em})\end{array}$ & $\begin{array}{l}\text { Interbedded sand } \\
\text { and gravel }\end{array}$ & $\begin{array}{l}\text { Discontinuous and diffuse } \\
\text { laminations; subplanar beds, } \\
\text { imbrications; lag deposits at lower } \\
\text { contact; poorly fossiliferous }\end{array}$ & $\begin{array}{l}\text { Coastal reworking; alluvial } \\
\text { traction currents; eolian } \\
\text { reworking }\end{array}$ & $\begin{array}{l}\text { Lies above De1, De2, } \\
\text { Pa2, Pa3 with an erosive } \\
\text { contact; forms the upper } \\
\text { unit }\end{array}$ & $\begin{aligned} 8180 & \pm 60 \\
\text { to } & \\
510 \pm & 70\end{aligned}$ & $\begin{array}{l}\text { Coastal reworking } \\
\text { (swash-and-backswash } \\
\text { and 'glaciel'); eolian } \\
\text { reworking }\end{array}$ \\
\hline
\end{tabular}


interlaminated sand, silt and silty clay and De1b consist of massive diamicts. Sand layers (De1a) located nearer to the former ice front are coarse and massive and interfinger with diamicts (De1b) while those located at relative distances from it are fine and stratified. They are interlayered with silty sand and silt and silty clay massive and laminated beds. General bed inclination result from westward flowing paleo-currents. Deposition of these facies was dominated by suspension-settling and submarine gravity flows. Ice rafted debris are also incorporated, but in a lesser proportion. Sediment mass-movement processes were very active in the Nastapoka Hills submarine fans, where steep slopes contributed largely to sediment instability. Dewatering structures are present in many exposures and suggest rapid sedimentation. Sediment deformation and semi-consolidation caused by glaciotectonism occur in S9. Exposures of ice-proximal deposits in S8, S9 and S43 are located near former grounding lines and S32, S39 and S44 are close to frontal moraines.

\section{Deglacial deposits: ice-distal sediments (De2)}

De2 sediments consist of interbedded and interlaminated silty clay and sand (Fig. 7a) and are, in some cases, similar to FA II silty clay. They form the lowermost unit in exposed sections. Silty clay is the predominant size fraction. Extensive zones of De2 occur in the inner basin below $200 \mathrm{~m}$ asl and are generally restricted to areas located along Nastapoka, Sheldrake and du Nord rivers. These deposits can be as much as $85 \mathrm{~m}$ thick in deep bedrock basins (Allard and Seguin, 1985). The considerable thickness of this unit in valleys of the shield necessarily result from an abundant and rapid glaciomarine sedimentation, considering the short period of only $300-400 \mathrm{yr}$ for the ice to retreat and the rapid emergence rate at deglaciation time. Such silty clay deposits are one of the most distinctive constituent of meltwater derived sediments in temperate and subpolar glaciomarine environments (Cowan and Powell, 1990). De2 deposits, exposed in S1a, S20 and $\mathrm{S} 45$, are in some cases deformed, showing wavy parallel and chaotic bedding and normal faults. Outsized clasts and fossil shells are sparsely distributed in this unit. Some layers of De2 are composed of blue-grey silty clay with a strong sulphur odour (Fig. 7b). These beds are varved and contain black speckles. The blue-grey color of this silty clay indicate a direct glacial input (Powell, 1983). Thin black layers are monosulphide layers (Elverhøi et al., 1980, 1983), which are typical in glaciomarine high accumulation rate conditions (Görlich, 1986). These layers were preserved by rapid sedimentation that limited the effect and rate of their transformation into pyrite and their metabolisation to organic matter (Berner, 1984; Stevens, 1990). They correspond to annual organic varves probably formed during a time-lag between the important spring diatom bloom and clastic summer sedimentation (Elverhøi et al., 1980). Textural and structural characteristics indicate that De2 sediments were transported by turbid meltwater plumes emanating from the glacial margin (hypopycnal flow) and were deposited by suspension-settling (Elverhøi et al., 1980, 1983; Powell, 1981; Görlich, 1986; Cowan and Powell, 1990). The presence of thin sand layers within silty clay beds may be associated with turbulence caused by shifting of the meltwater jet (McCabe et al., 1993) or by submarine gravity flows. In S1a, sand beds associated with De1 sediments occur above older ice-distal De2 laminated silty clay. This can be explained by a remobilization of the original ice-proximal sand deposition and by their progradation on silty clay.

\section{Paraglacial deposits $(\mathrm{Pa})$}

Paraglacial sediments $(\mathrm{Pa})$ are coarse-grained fluvial deposits transported to the marine environment under conditions of abnormally high transport rates associated with the rapid ablation of ice sheets in the hinterland (Church and Ryder, 1972; Syvitski et al., 1987, Syvitski, 1991). During this period, sediment yields were high due to fluvial-related erosion and transportation of large volumes of pre-deposited glacigenic and marine sediments (Syvitski, 1991). In the study area, Pa sediments were deposited as the glacial margin was retreating eastward towards central Québec-Labrador, inland from the marine limit. Pa deposits form extensive zones of thick sand bodies on the lower western slopes of the Nastapoka Hills. They are concentrated near the Nastapoka, Sheldrake and Umiujaq river mouths, where they are exposed between 0 and $100 \mathrm{~m}$ asl. They are considerably more extensive near the Rivière Nastapoka mouth. Pa deposits consist of well sorted and bedded sand bodies associated with fan deltas and comprise from base to top three members: 1 ) interbedded and interlaminated silty clay and sand (Pa1);2) interbedded sand, silt and silty clay (Pa2); and 3) laminated and massive well sorted fine to medium sand beds ( $\mathrm{Pa} 3$ ).

Pa1 sediments form sequences of massive and laminated silty clay irregularly alternating with laminated and massive fine sand, silty clay being the predominant member. They are well exposed and more concentrated near the Nastapoka (S2, S3) and Umiujaq (S41) river mouths. These sediments overlie ice-distal beds with unclear contacts. They are easily distinguishable from ice-distal sediments by their much higher sand composition and concentrations of sand laminations. This unit is located at relatively lower altitudes ( $<53 \mathrm{~m}$ asl) and underlies $\mathrm{Pa} 2$ rhythmic beds with a gradation contact. However, in S41, they are found in sharp contact with Pa3. Contacts between Pa silty clay and sand beds (Fig. 7c) are sharp, erosive or gradational. Pa1 were deposited during periods of predominant suspension settling and bedload. Variations in overall relative bed thickness of fines and sand is attributed to changes of the sediment source. Increasing thickness of sand beds in the upper parts of this unit corresponds to increasing proximity of the sediment source and higher energy due to glacioisostatic uplift. The muddy texture and the position of $\mathrm{Pa} 1$ below $\mathrm{Pa} 2$ rhythmic beds suggest that they are bottomsets. Terrestrial organic matter and wood fragments occur in the deposits.

$\mathrm{Pa} 2$ sediments are composed of rhythmically interbedded well sorted to poorly sorted fine sand and poorly sorted silty sand and/or poorly sorted silty clay (Fig. $7 \mathrm{~d}$ ). They are restricted to relatively low elevations $(<70 \mathrm{~m}$ asl) and are mainly concentrated near the Nastapoka (S2-S5), Devaux (S37) and Umiujaq (S41) river mouths. They overlie Pa1 beds with sharp or gradation upper contact. In areas unaffected by erosion, $\mathrm{Pa} 2$ beds always underlie $\mathrm{Pa} 3$ beds. They generally 


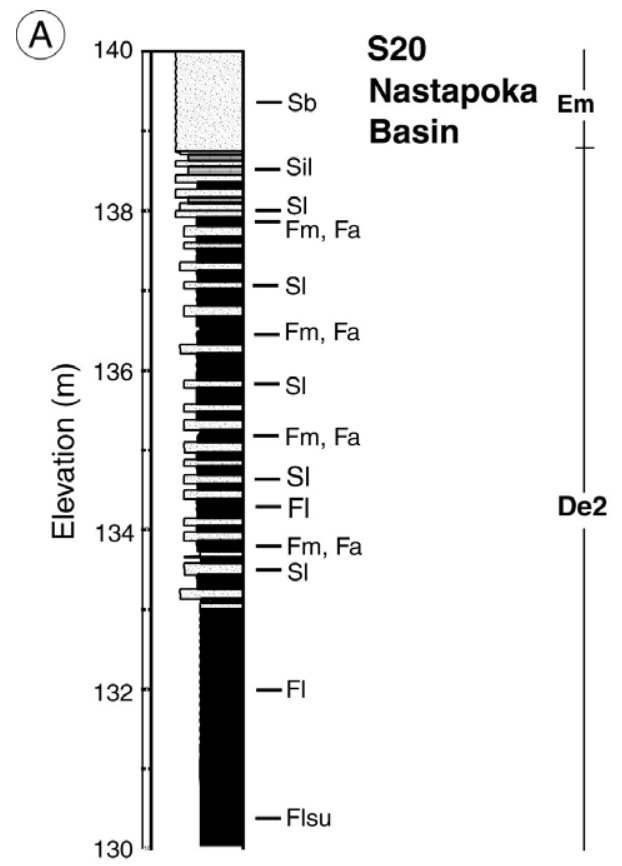

FIGURE 6. Representative logs of cross-sections through ice-proximal (De1), ice-distal (De2), paraglacial $(\mathrm{Pa})$ and emergence (Em) lithofacies associations, Rivière Nastapoka.

present a slight westward inclination. The rhythmites resemble closely to varves. Sand beds become progressively thicker and more numerous than the silty sand and silty clay beds in the upper parts of this unit. These deposits contain no fossil shells and are usually not bioturbated, although ichnofossils were observed in some exposures. Processes responsible for deposition of $\mathrm{Pa} 2$ are suspension, bedload and recurrent small-scale gravity flow. A coarsening-upward and gradation upper and lower contacts indicate progradation due to glacioisostatic uplift. Rhythmic bedding of fines with sand found in these beds are typical of a prodeltaic environment and are interpreted here as transitional deposits between foresets and bottomsets. The generally rare bioturbations suggests rapid sedimentation.

Pa3 are composed of laminated and massive well sorted fine to medium sand (Fig. 7e). They occur at elevations lower than $100 \mathrm{~m}$ asl near the Nastapoka (S3-S5), Devaux (S36, S37) and Umiujaq (S41) river mouths. These beds form the main body of a very large raised delta $63 \mathrm{~m}$ asl near the Rivière Nastapoka mouth. $\mathrm{Pa} 3$ reaches maximum thickness of $16 \mathrm{~m}$ in S5. Coarser sand and gravel beds occur in this unit and usually tend to be poorly sorted. Laminated beds are generally inclined $\left(25^{\circ}\right)$ and oriented WNW. Inclined $\left(25^{\circ}\right)$ and WNW oriented $<20 \mathrm{~cm}$ thick rhythmically interbedded sand and silt of $\mathrm{Pa} 2$ occur with sharp contacts within these deposits. $\mathrm{Pa} 3$ beds overlie $\mathrm{Pa} 2$ beds with gradation or sharp contacts.

\begin{tabular}{|c|c|c|c|}
\hline & & LEGE & \\
\hline & & Code & Lithofacies type \\
\hline 5 & Diamict & $\mathrm{Dmm}$ & Massive, matrix supported \\
\hline & Gravel & $\mathrm{Gm}$ & Massive to crudely bedded \\
\hline & & Gp & Planar cross-beds \\
\hline & Sand & $\mathrm{SI}$ & Laminated \\
\hline & & $\mathrm{Sm}$ & Massive \\
\hline & & $\mathrm{Sg}$ & Graded \\
\hline & & $\mathrm{Sr}$ & Current ripples \\
\hline & & $\mathrm{Sp}$ & Planar cross-beds \\
\hline & & St & Through cross-beds \\
\hline & & Sdl & Diffusely laminated \\
\hline & & Sd & Deformed \\
\hline & Silt & Sil & Laminated \\
\hline & & Sim & Massive \\
\hline & & Sig & Graded \\
\hline & & Sir & Current ripples \\
\hline & & Sid & Deformed \\
\hline & Silty clay & $\mathrm{FI}$ & Laminated \\
\hline & & $\mathrm{Fm}$ & Massive \\
\hline & & $\mathrm{Fa}$ & Agregated \\
\hline & & Flsu & Laminated, mono-sulphide layers \\
\hline & & $\mathrm{Fd}$ & Deformed \\
\hline & & & (After Eyles et al., 1983) \\
\hline
\end{tabular}

Coupes stratigraphiques représentatives dans des sédiments glaciaires proximaux (De1), distaux (De2), paraglaciaires $(\mathrm{Pa})$ et d'émersion $(E m)$, dans la rivière Nastapoka.

Upper contacts of $\mathrm{Pa} 3$ with emergence beds are erosive or scoured. The high-angle dipping, prograding sand and occasional gravel beds of $\mathrm{Pa} 3$ sediments are characteristics of foresets. Paleocurrent data also suggest a sediment source in the coastal hills flowing towards the Hudson Bay coastline, which is similar to modern river flow.

\section{Emergence deposits (Em)}

Emergence deposits were laid in place in a wave affected environment when the glaciomarine and paraglacial sediments were exposed to coastal processes during emergence. Em consists of interbedded sand and gravel (Fig. 7f). They are exposed in almost all the studied sections where they form the uppermost unit. They tend to become thicker at lower elevations. Em are composed of well to poorly sorted massive to crudely bedded gravel, granules and coarse sand alternating with well to poorly sorted laminated and massive coarse to fine sand. They are generally $0.75-2 \mathrm{~m}$ thick but can vary from a few $\mathrm{cm}$ to $5 \mathrm{~m}$ thick. Lower contacts are erosive and scoured and often form lag deposits. Presence of cross-bedding, planeparallel lamination and gravel imbrications indicate bottomcurrent processes. Discontinuous and wavy sand laminations, frequent heavy mineral layers and very good sorting are typical of beach deposits. Swash-and-backswash and ice rafting processes disturbed, eroded and re-transported previous laid 
(B)

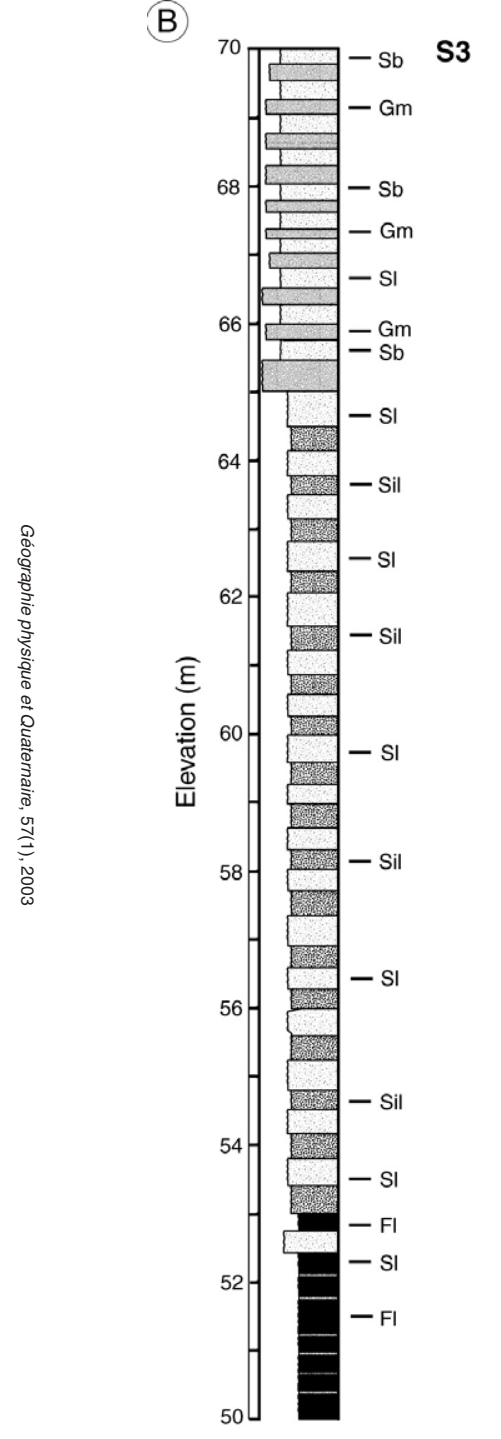

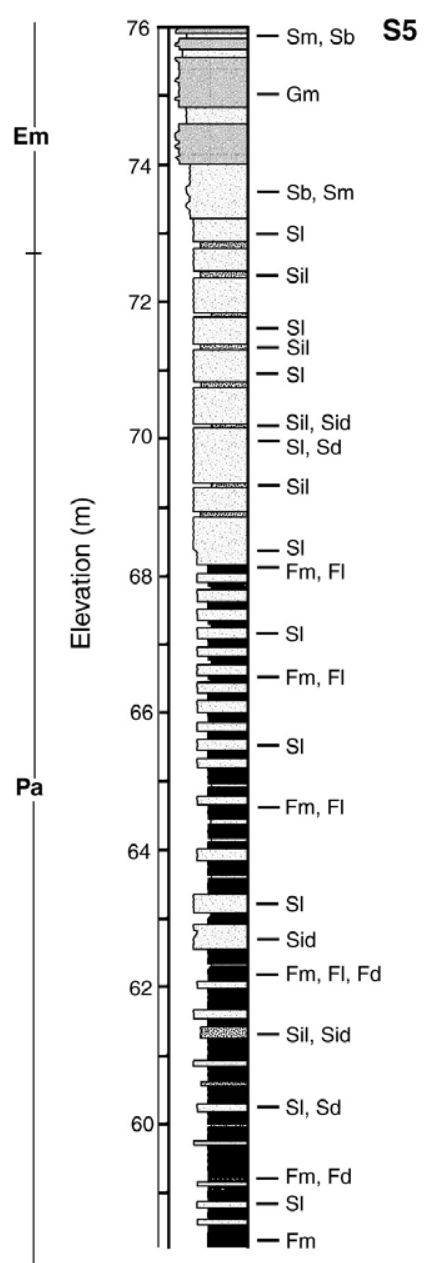
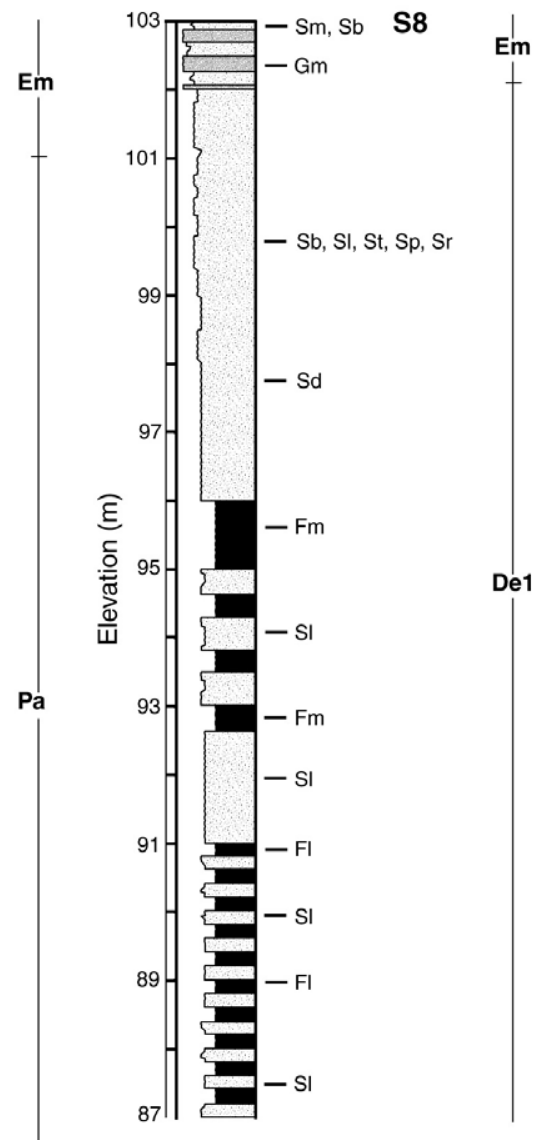

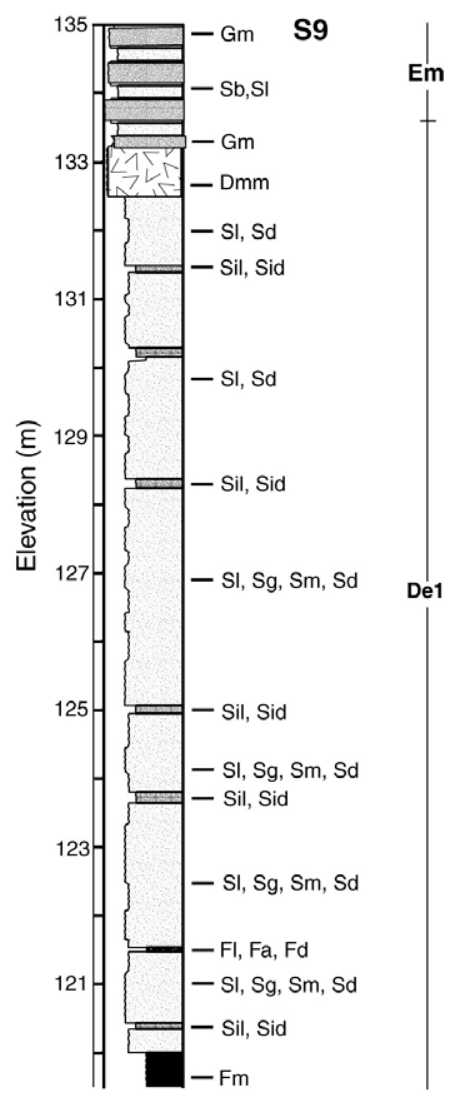



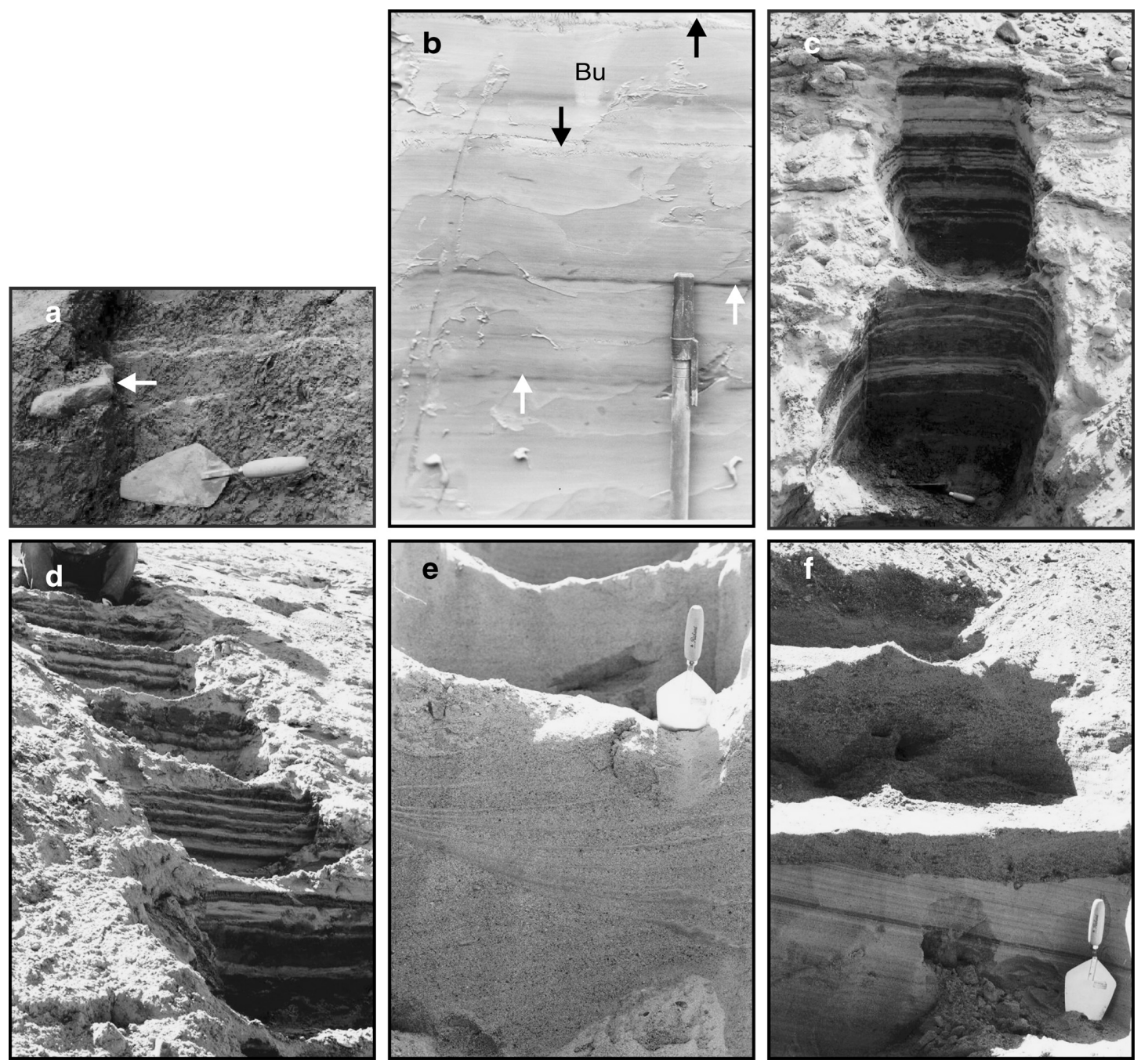

FIGURE 7. a) Massive silty clay bed with undulating fine sand lamination and IRD (De1a); b) Blue-grey silty clay containing black monosulphide layers (white arrows) with sand laminations (black arrows) in $a$ De2b bed. Bioturbations occur in these sediments, such as burrows (Bu). Black or greyish speckles are also attributed to bioturbations; c) Interbedded and interlaminated silty clay and sand (Pa1) (photo: courtesy of Denis Sarrazin); d) Rhythmically interbedded, silty-sand and silty-clay (Pa2); e) Channel fill structure in $\mathrm{Pa} 3$; f) Erosive contact between $\mathrm{Pa} 3$ and $\mathrm{Em}$ deposits. a) Lit de silt argileux massif et sable fin laminé avec bloc délesté par les glaces flottantes (De1a); b) silt argileux bleu-gris contenant des lits de monosulphides noirs (De2b) (la présence de taches noires et grises est aussi attribuée à des bioturbations) ; c) silt argileux et sable interlités et interlaminés (Pa1) (photo de Denis Sarrazin) ; d) rythmites de sable silteux et silt argileux (Pa2); e) structures à chenaux dans $\mathrm{Pa} 3$; f) contact d'érosion entre Pa3 et Em. 
deposits, thus affecting their original character. Presence of under-consolidated very fine sand and diffuse laminations in the upper parts of this unit is attributed to some eolian reworking that most likely started in the beach environment. This process formed dunes on the surface of raised sandy accumulations. Lulls in eolian activity lead to development of soils. Paleosoils were preserved by the return of eolian activity (Filion et al., 1991; Saint-Laurent and Filion, 1992) and provided minimum ages for sediment emergence.

\section{CHRONOLOGY}

A great part of the radiocarbon dated material was recovered in exposures located near the Rivière Nastapoka mouth (Tabl. I; Fig. 3). The oldest radiocarbon dates are concentrated along the Nastapoka Hills and provide minimum ages for deglaciation. Many of these dates were recovered in De1a, in many cases associated with submarine gravity flows lithofacies. An age of 8580 cal. yr BP (UL-2346; S47) was obtained on paired valves Macoma balthica at $4 \mathrm{~m}$ asl in De2b sediments. This sample, recovered in ice-distal glaciomarine sediments deposited during the Nastapoka Hills stillstand, yielded the oldest radiocarbon date of the study area. However, since $\Delta R$ varies greatly in eastern Hudson Bay, reaching in some cases exceptionally high values of nearly 600 years (Barber et al., 1999), it is possible that sample UL-2346 could overestimate by a few hundred years the real age of the Nastapoka stillstand. In order to present a more cautious interpretation of deglacial events in the sector, this sample is disregarded in this study, even if its age is still enclosed within the extremes of the $1 \sigma$ age range for the sudden drainage event of Glacial Lake Ojibway of 8160-8740 cal. yr BP (Barber et al., 1999). A younger age of $8300 \mathrm{cal}$. yr BP was provided by in situ Mya truncata, Hiatella arctica and Macoma balthica recovered $1 \mathrm{~km}$ downstream from the upper terrace of an ice-contact submarine fan at $213 \mathrm{~m}$ asl (UL-1932; S29; $145 \mathrm{~m}$ asl). Although the age of this sample could also slightly overestimate the timing of deglaciation due to a possible abnormally high $\Delta R$ value, it is contemporaneous with the $8280 \mathrm{cal}$. yr BP age for the drainage of Glacial Lake Ojibway reported in Barber et al. (1999) for southeastern Hudson Bay. UL-1932 is thus used to date deglaciation and as a minimal age for the $248 \mathrm{~m}$ marine limit in the Rivière Nastapoka area. This age also supports the model of a contemporaneous ice-stillstand in southeastern Hudson Bay and eastern James Bay reported by Lajeunesse and Allard (2003). In the same section, $9 \mathrm{~m}$ above UL-1932, a younger age of $7840 \mathrm{cal}$. yr BP was provided by a sample of in situ Mya truncata and Macoma balthica (TO-7411; S29; $154 \mathrm{~m}$ ). A slightly older age of 7920 cal. yr BP (UL-1951; S19) was yielded by valves of Hiatella arctica at $108 \mathrm{~m}$ asl in a massive silty sand bed of De1a associated with another fan exposed along Stream A. An age of $8180 \mathrm{cal}$. yr BP was yielded by a driftwood sample recovered in $\mathrm{Em}$ sediments at $5 \mathrm{~m}$ asl along the shoreline (CAMS-37437; S10). This sample was recovered in modern sediments and in association with a sample of Chlamys islandicus that gave an age of 4630 cal. yr BP (CAMS-37434; S10). A few hundred metres south of Rivière Nastapoka, in the hill range area, valves and fragments of Hiatella arctica found on the surface of mudboils
(De2a) gave an age of 7750 cal. yr BP (UL-1740; S45; $115 \mathrm{~m}$ ). This sample recovered in ice-distal deposits slightly east of the hills indicate that the Nastapoka Hills were already deglaciated by $7.8 \mathrm{ka}$ cal. BP.

Fossil shells recovered in highly deformed sediments provide maximum and minimum ages for submarine gravity flow events. Fragments of Hiatella arctica from De1a disturbed laminated sand at $110 \mathrm{~m}$ asl exposed along a stream near Rivière Nastapoka gave an age of 7700 cal. yr BP (UL-1728; S7). Fragments of Mytilus edulis and Hiatella arctica found at $132 \mathrm{~m}$ asl in De1 silty clay exposed along Stream B yielded an age of 7500 cal. yr BP (UL-1722; S27). In similar deposits of the same section, in situ Hiatella arctica and Mytilus edulis gave an age of 7360 cal. yr BP (UL-1718; $112 \mathrm{~m})$. Near Rivière Devaux, fragments of Hiatella arctica and Mytilus edulis found in an exposure perched along a steep valley wall in De1a at $146 \mathrm{~m}$ asl gave an age of $7130 \mathrm{cal}$. yr BP (Beta-121551; S40). In "Vallée-des-Trois", near Lac Guillaume-Delisle, fragmented Hiatella arctica and Mytilus edulis at $100 \mathrm{~m}$ asl in an exposure of De1b diamict bed deposited by submarine mass movement dated 7020 cal. yr BP (Beta-125152; S43). Younger radiocarbon dates in similar deposits were provided by paired valves of Mya truncata at $109 \mathrm{~m}$ asl that dated 6530 cal. yr BP (TO-7409; S13) and paired valves of Mytilus edulis and Hiatella arctica at $103 \mathrm{~m}$ asl that dated $6570 \mathrm{cal}$. yr BP (UL-1710; S17). Nearby $\mathrm{S} 17$, valves of Hiatella arctica in highly disturbed De1 laminated sand at $97 \mathrm{~m}$ asl dated 6660 cal. yr BP (UL-1540; S23). The same age was provided by a sample collected by Allard and Seguin (1985) at $118 \mathrm{~m}$ asl in similar deposits near S23 (UQ-540). Fragments of remobilized Hiatella arctica and Mytilus edulis in a silty sand layer at $84 \mathrm{~m}$ asl gave an age of 6730 cal. yr BP (UL-1952; S38). This sample provides a maximum age for a submarine landslide that occurred in a high RSL. The radiocarbon database from De1 disturbed beds suggests that submarine mass-movements were still active in the submarine fans about $6.7 \mathrm{ka}$ cal. BP, some 1500 years after their deposition in shallowing marine conditions.

Submerged driftwood collected at $51 \mathrm{~m}$ in Pa1 deltaic sediments near the Rivière Nastapoka mouth dated 5760 cal. yr BP (UL-1556; S3). The upper terrace of the delta is located at $\sim 100 \mathrm{~m}$ asl. UL-1556 therefore provides a maximum age of $5.8 \mathrm{ka}$ cal. BP for the formation of this delta terrace. Near the Rivière Devaux mouth, fragments and valves of mixed shells at $15 \mathrm{~m}$ asl in $\mathrm{Pa} 3$ gravel dated 4340 cal. yr BP (UL-1919; S36) and at $13 \mathrm{~m}$ asl in similar sediments dated 3870 cal. yr BP (UL-1917; S36). The stratigraphic position of UL-1917 under $10 \mathrm{~m}$ of sediments implies that an important sedimentation was still prevailing in relatively shallow water offshore after $3.9 \mathrm{ka}$ cal. BP. An age of $3550 \mathrm{cal}$. yr BP (UL-1546; S1a) was yielded by undifferentiated shells fragments in Em alluvial bedded sand at $12 \mathrm{~m}$ asl. Paired valves of Hiatella arctica recovered at a lower elevation east of S43 in Pa2 sediments dated 3040 cal. yr BP (UL-1371).

Organic samples of buried paleosoils were collected at high elevations near the Nastapoka Hills. Paleosoil samples UL-1904, at $98 \mathrm{~m}$ asl, and UL-1878, at $136 \mathrm{~m}$ asl, gave ages of 5420 cal. yr BP (S14) and 4750 cal. yr BP (S46), respectively. These samples provide minimum ages for emergence of 
their enclosing sediments. Paleosoil samples UL-1903 (1960 cal. yr BP; S24; $150 \mathrm{~m}$ asl) and UL-1949 (730 cal. yr BP; S9; $133 \mathrm{~m}$ asl) developed long after emergence and are not representative of land uplift. However, a younger paleosoil sampled in the trough of an ice-wedge (polygon) at $12 \mathrm{~m}$ asl near the shore gave an age of 310 cal. yr BP (UL-1858; S1b) and provides a minimum age of emergence of the coastal beach gravel $(\mathrm{Em})$ in which it developed. Two driftwood samples collected at $4 \mathrm{~m}$ asl under $\sim 40-60 \mathrm{~cm}$ of beach sand (Em) in the vicinity of $S 1 \mathrm{~b}$ yielded an age of $510 \mathrm{cal}$. yr BP (UL-1536; UL-1528), providing a minimal age for emergence of the deposits. Basal peat and wood samples recovered at $170 \mathrm{~m}$ asl over De2 sediments east of the Nastapoka Hills south of Rivière Nastapoka dated 6640 cal. yr BP (I-13130 and I-13131) (Allard and Seguin, 1985). Another sample collected by the same authors at Lac Sheldrake at $180 \mathrm{~m}$ asl dated $6910 \mathrm{cal}$. yr BP (I-13406), indicating that the underlying sediments were emerged by $6.9 \mathrm{ka}$ cal. BP.

\section{DISCUSSION}

\section{LAND UPLIFT AND EMERGENCE CURVE}

Fossil shells and driftwood buried under marine or coastal sediments provide maximum ages for the emergence of their overlying material whereas paleosoils, wood and peat buried by eolian processes or organic accumulation provide minimum ages for emergence. Hence, radiocarbon ages on shells and driftwood are located below the RSL curve while those from paleosoils, terrestrial wood and peat are above it. The assemblages of fossil shells recovered are not typical of the shore zone and the emergence facies are not fossiliferous; therefore no single raised beach or shoreline could be dated, such as what was done on the Manitounuk Islands, south of the study area (Allard and Tremblay, 1983a). The available data allows only a constrained RSL curve to be deducted (Fig. 8).

The RSL curve is characterized by an inflection between 5 and $6 \mathrm{ka}$ cal. BP. This inflection indicates that the emergence occurred in an abruptly decelerating mode and divides the curve into three phases: 1) before $6 \mathrm{ka}$ cal. BP; 2) between 6 and $5 \mathrm{ka}$ cal. BP; and 3) after $5 \mathrm{ka}$ cal. BP. Most fossils shells reported in this study were recovered in sediments deposited or remobilized by submarine mass movements before $6.5 \mathrm{ka}$ cal. BP. A very important part of the $248 \mathrm{~m}$ total Holocene emergence took place in the first phase of glacioisostatic rebound.

After reaching a maximum elevation of $248 \mathrm{~m}$ asl about $8.3 \mathrm{ka}$ cal. BP in the Nastapoka Hills, the Tyrrell Sea regressed rapidly at a rate of $\sim 7 \mathrm{~m} / 100 \mathrm{yr}$ during deglaciation. This initial uplift rate is similar to the previously reported maximum rate of $6 \mathrm{~m} / 100 \mathrm{yr}$ on Ottawa Islands by Andrews and Falconer (1969). This emergence was accompanied by extensive and

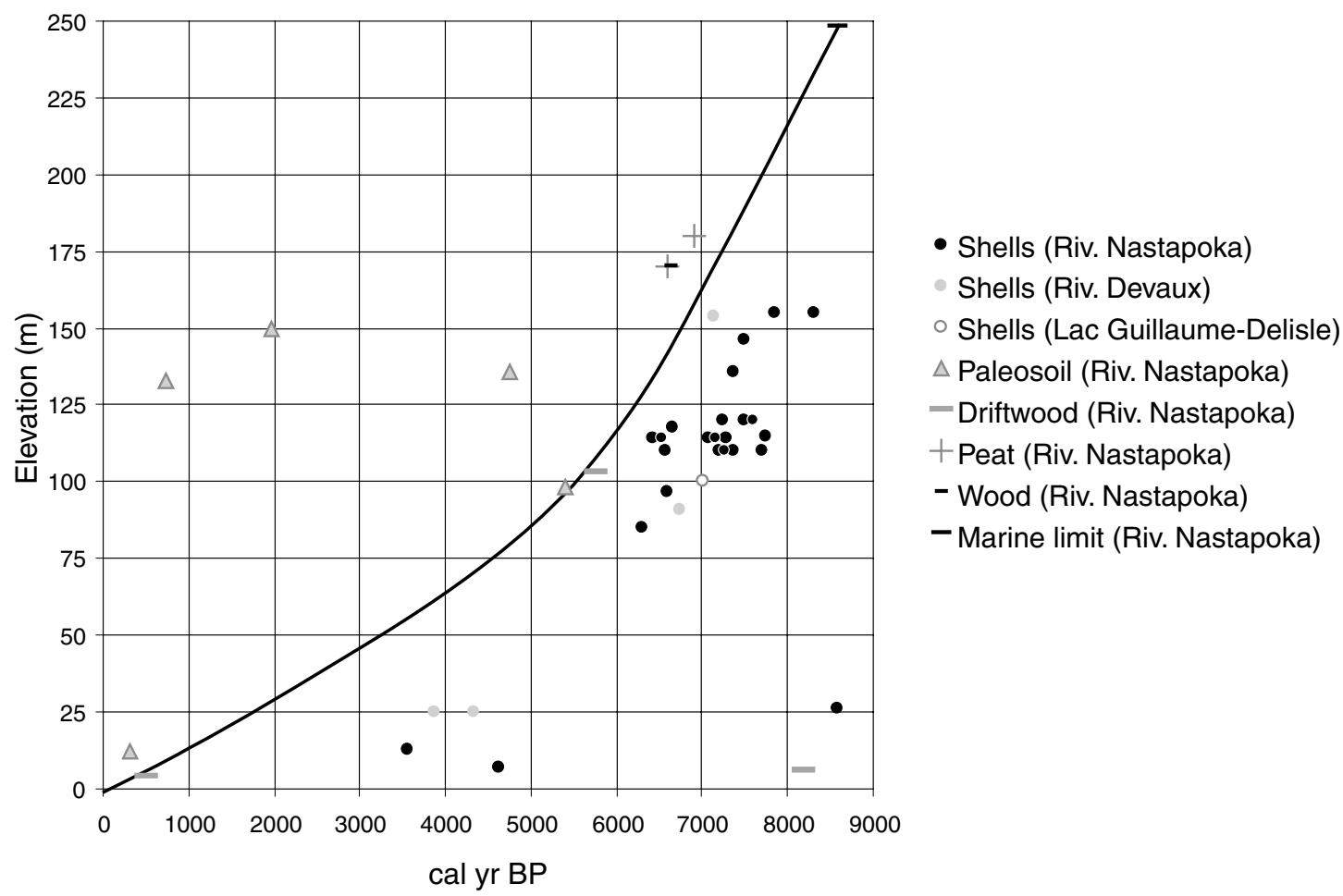

FIGURE 8. Postglacial relative sea-level curve of the Rivière Nastapoka area, eastern Hudson Bay.

Courbe du niveau marin relatif postglaciaire de la région de la rivière Nastapoka, sur la côte est de la baie d'Hudson. 
numerous submarine gravity flows on the steep slopes of icecontact submarine fans located on the structural slope west of the Nastapoka Hills. These events cannot be directly attributed to postglacial seismicity linked to glacioisostatic recovery since they occurred in a setting prone to mass-movements (Lajeunesse and Allard, 2002). Slope instabilities in soft sediments, possibly triggered by bottom wave disturbances in shallowing waters might have been a key factor for submarine landslides during this phase. Following this period of homogeneous uplift rates (upper segment of the RSL curve), emergence decelerated to reach rates of $\sim 4 \mathrm{~m} / 100 \mathrm{yr}$ between 6 and $5 \mathrm{ka}$ cal. BP (inflection segment of the curve). This phase was characterized by a period of vegetation development on emerged sediments, in accordance with the timing of forest-tundra colonisation inland (Payette, 1983). Since $5 \mathrm{ka}$ cal. BP, $\sim 85 \mathrm{~m}$ of uplift has occurred at rates of $\sim 1.7 \mathrm{~m} / 100 \mathrm{yr}$ (lower segment of the curve). Our data suggest an emergence rate slightly slowing down to $\sim 1.6 \mathrm{~m} / 100 \mathrm{yr}$ over the last 1000 years, which is higher that the $1.3-1.5 \mathrm{~cm} / \mathrm{yr}$ at Manitounouk Islands (Allard and Tremblay, 1983a; Bégin et al., 1993), $1.4 \mathrm{~cm} / \mathrm{yr}$ near Inukjuak (Gray et al., 1993) and $1.1 \mathrm{~cm} / \mathrm{yr}$ at Lac Guillaume-Delisle (Hillaire-Marcel, 1976). However, more dates are needed on this segment of the curve in order to improve the resolution of the recent uplift rate.

Land emergence led to extensive incision by rivers and streams through sand and silty clay deposits, which remobilized and carried downstream important volumes of sediments. RSL fall exposed marine sediments deposited during the deglaciation to the action of coastal processes. Exposed bedrock outcrops were disrupted by gelifraction; ensuing large rock fragments were redistributed by drift ice and added to glacial erratics to form boulder pavements and ridges. Accumulations of sand deposits were reworked by swashand-backswash and eolian processes to form successive beach ridges. The upper portion of emergence deposits was reworked by eolian processes that formed dunes and deflation surfaces. During the late phases of emergence, the sedimentary regime of the Rivière Nastapoka was strongly affected by the formation of two important falls near the hill range. These bedrock sills formed inland basins that restrained coarse material from reaching the seashore and reduced greatly sand transport to the Rivière Nastapoka mouth (Lavoie, 2000; Lavoie et al., 2002). Ice-distal glaciomarine silty clay (De2), which was previously buried under deltaic deposits, will probably be the predominant sediment emerging in the future.

Considering the very important postglacial emergence of the area $(\sim 250 \mathrm{~m})$ and the very high rates of regression still prevailing today ( $8.3 \mathrm{ka}$ cal. BP following deglaciation), it is questionable if this uplift can be attributed totally to glacioisostasy. In fact, an abnormally cold and dense downwelling convective region in the earth's mantle located below the North American craton (Mitrovica, 1997; Simons and Hager, 1997) may have partially acted to depress the earth's crust and/or contributed to create a sensitive zone for isostatic depression during the last glaciation.

\section{DEGLACIATION AND SEDIMENTATION}

Geomorphic, sedimentary and radiometric data indicate that deglaciation of the Rivière Nastapoka area was marked by four phases. During Phase 1, an ice stillstand occurred at $8.3 \mathrm{ka}$ cal. BP along the Nastapoka Hills after a period of very rapid down-wasting and collapse over Hudson Bay (Lajeunesse and Allard, 2003). Large ice-contact glaciomarine fans were laid from the hills on the sea bottom, where now extends the coastal slope. At that time, theTyrrell Sea was at its maximum elevation and the emergence rate was extremely rapid. Sedimentation was dominated by suspension-settling from turbid glacial meltwater outflows, submarine gravity flows and ice rafting (Lajeunesse and Allard, 2002).

Phase 2 was marked by the unpinning of the ice front from the Nastapoka Hills and its rapid eastward retreat in the low elevation inner basin to the easternmost reaches of the Tyrrell Sea, about $35 \mathrm{~km}$ inland. This distance, together with the rate of retreat of $108 \mathrm{~m} / \mathrm{yr}$ suggested by the DeGeer moraines, indicate that this phase lasted for 330 years. At the end of this phase, RSL had dropped to about 200-190 m asl. The high RSL in this deep basin exerted an important control on the glacial margin by promoting high rates of calving and fast ice flow and thus favouring ice sheet collapse and drawdown (e.g., Hugues, 1987; McCabe et al., 1993). Sedimentation was dominated by glacial meltwater outflows that deposited a thick silty clay drape from suspension-settling in submerged fjord-like valleys. During this period, the ice-contact glaciomarine fans of the Nastapoka Hills complex were intensively affected by submarine landslides. Timing control of this phase from radiocarbon dates is needed. However, considering that the end of Phase 2 sedimentation occurred in a RSL of $\sim 200-190 \mathrm{~m}$, it can be deducted from the uplift curve (Fig. 8) that this phase occurred prior to $\sim 7.5 \mathrm{ka}$ cal. BP.

Phase 3 was characterized by a second temporary pause or slowdown in ice retreat at the eastern margin of the Tyrrell Sea invasion, where elevations are slightly higher than in the inner basin. This phase began when RSL was still $\sim 200-190 \mathrm{~m}$ asl, i.e. prior to $\sim 7.5 \mathrm{ka}$ cal. BP. The topography of this part of the study area and the rapid RSL fall caused this stabilisation by allowing the ice margin to become terrestrial, thus considerably reducing its ablation rate in shallower marine waters at the terminus. This phase lead to the deposition of a second regional drift belt consisting of sandur-deltas in association with important esker systems characterized by the same direction of flow than major rivers. Deposition of this sediment belt corresponds to either a complete stillstand of the ice margin or a change in sedimentation regime when the ice margin changed from a tidewater to a terrestrial environment. Sedimentation was dominated by glaciofluvial processes on the surface of sandurs and by turbid plumes in their submerged zones that deposited ice-proximal sand and silt. These plumes transported fines westward from these glaciofluvial systems and deposited silty clay along the fjord-like bedrock basins. According to shells recovered in De2 east of the Nastapoka Hills, ice-distal sedimentation continued until at least 7240 cal. yr BP (UQ-545). There is no evidence in support of a complete belt of ice recession for a set period on the Hudsonian Plateau. However, some far inland sectors were ice free at $6.6 \mathrm{ka}$ cal. BP since organic remains had by then begun to accumulate in alluvial deposits over clay in the Rivière Sheldrake basin (Allard and Seguin, 1985). This phase was also early characterized by the development of a discontinuous 
permafrost (Allard and Seguin, 1987) and periglacial landforms (Lajeunesse, 2000).

Phase 4 was associated with the complete retreat of the ice margin from the area and its terrestrial ablation inland. Although the ice terminus was distant during this phase, sedimentation dynamics were still controlled by meltwaters. Sedimentation was dominated by fluvial processes that transported large amounts of glacigenic material along major river systems, which is typical of paraglacial sedimentary environments (Church and Ryder, 1972; Syvitski, 1993). During this period, sediment delivery was essentially controlled by river downcutting through pre-existing emerged deposits. Within the inner basin and where silty clay deposits are present, elevations of the third segment of the Rivière Nastapoka upstream from the second falls are between 125 and $160 \mathrm{~m}$ asl. This implies that the RSL was probably $<160 \mathrm{~m}$ asl, considering that paraglacial deposits do not occur along the upper sections of Rivière Nastapoka. At the mouth of the paleo-Rivière Nastapoka, a thick and extensive fan delta developed $<100 \mathrm{~m}$ asl. The RSL fall also lead to the incision, erosion and remobilization of ice-proximal and ice-distal glaciomarine and glacial sediments located along the fluvial system. These sediments were transported and deposited near the actual Rivière Nastapoka mouth as well as near other less important river mouths when the ice margin was tens and probably hundreds of kilometres distant. According to wood fragments (UL-1556) recovered in a fossil rich Pa1 bed, paraglacial sedimentation had already begun in some valleys by $5760 \mathrm{cal}$. yr BP. The fact that this rich fossil bed is overlain by more than $19 \mathrm{~m}$ of deposits points out abnormally high sedimentation rates that prevailed during this period, which is not representative of typical postglacial sedimentation conditions in Hudson Bay (Josenhans et al., 1988). This considerable sedimentation is attributed to the disintegration of Québec-Labrador ice and sediment reworking. Deposits similar to those reported here have been observed near the mouth of Grande Rivière de la Baleine (Gonthier et al., 1993).

\section{CONCLUSION}

This study integrating geomorphological, sedimentary, stratigraphic and radiometric data presents new information on the style and timing of deglaciation and glaciomarine deposition, and provides an additional uplift curve for eastern Hudson Bay. In the study area, deglaciation and sedimentation were controlled by topography and RSL fall and were marked by four phases: 1) a stillstand of the Québec-Labrador ice margin at $8.3 \mathrm{ka}$ cal. BP along the Nastapoka Hills that deposited a belt of ice-contact glaciomarine sediments. During this phase, RSL was high and was falling very rapidly; 2 ) the unpinning of the margin from the hills followed by a retreat phase during a still rapidly falling RSL that deposited a thick drape of fine-grained glaciomarine sediments. DeGeer moraines suggest that this retreat took place in about 330 years; 3) a second stillstand along the margin of the higher relief of the Hudsonian Plateau which occurred when RSL had fallen to $\sim 2$ ka cal. BP. It deposited ice-proximal sandy sediments in sandur-deltas as well as ice-distal silty-clay in submerged valleys; and 4) a complete retreat of the Québec-Labrador ice from the study area at its disintegration further inland. This event generated meltwaters that eroded and transported important volumes of sediments along the coast in a decelerating fall of RSL. Paraglacial sedimentation along the coast of Hudson Bay was already underway at $5.8 \mathrm{ka}$ cal BP. Further investigations are needed in the area in order to evaluate the timing and duration of the second stillstand or retreat slowdown phase along the Hudsonian Plateau (Phase 3) and the rates of retreat toward central Québec-Labrador (Phase 4).

\section{ACKNOWLEDGEMENTS}

This research project was funded by FCAR (Fonds pour la formation de chercheurs et l'aide à la recherche, Gouvernement du Québec) through a Ph.D. Scholarship to Patrick Lajeunesse and operating grants to Michel Allard's team; and by NSERC (Natural Science and Engineering Research Council). Fieldwork was partly supported by the Department of Indian and Northern Affairs through an NSTP (Northern Scientist Training Project) grant. Joshua Sala, Denis Sarrazin and Caroline Lavoie assisted during the 1996, 1997, and 1998 field seasons. Caroline Lavoie assisted in drawing the figures. Jean-Claude Dionne (Université Laval), Bernard Hétu (Université du Québec à Rimouski), Jean Veillette (Geological Survey of Canada), and Caroline Lavoie provided helpful reviews of the manuscript. We also thank Serge Occhietti and John Shaw for their formal reviews that improved the quality of the manuscript.

\section{REFERENCES}

Allard, M. and Seguin, M.K., 1985. La déglaciation d'une partie du versant hudsonien des rivières Nastapoka, Sheldrake et à l'Eau Claire. Géographie physique et Quaternaire, 39: 13-24.

1987. The Holocene evolution of permafrost near tree-line on the eastern coast of Hudson Bay (northern Québec). Canadian Journal of Earth Sciences, 24: 2206-2222.

Allard, M. and Tremblay, G., 1983a. La dynamique littorale des îles Manitounuk durant l'Holocène. Zeitschrift für Geomorphologie, N.F. Suppl., 47: 61-95.

1983b. Les processus d'érosion littorale périglaciaire de la région de Poste-de-la-Baleine et des îles Manitounuk sur la côte est de la mer d'Hudson, Canada. Zeitschrift für Geomorphologie, N.F. Suppl., 47: 27-60.

Andrews, J.T. and Falconer, G., 1969. Late glacial and post-glacial history and emergence of the Ottawa Islands, Hudson Bay, N.W.T.: Evidence on the deglaciation of Hudson Bay. Canadian Journal of Earth Sciences, 6: 1283-1276.

Avramtchev, L., 1982. Catalogue des gîtes minéraux du Québec, région de la Baie d'Hudson, Québec. Ministère de l'Énergie et des Ressources, Service de la géoinformation, Québec, DPV-926, 13 p.

Barber, D.C., Dyke, A., Hillaire-Marcel, C., Jennins, A.E., Andrews, J.T., Kerwin, M.W., Bilodeau, G., McNeely, R., Southon, J., Morehead, M.D. and Gagnon, J.-M., 1999. Forcing of the cold event of 8,200 years ago by catastrophic drainage of Laurentide lakes. Nature, 400: 344-348.

Bégin, Y., Bérubé, D. and Grégoire, M., 1993. Downward migration of coastal conifers as a response to recent land emergence in Hudson Bay, Québec. Quaternary Research, 40: 81-88.

Berner, A., 1984. Sedimentary pyrite formation: An update. Geochemica and Cosmochimica Acta, 48: 605-615.

Bilodeau, G., de Vernal, A., Hillaire-Marcel, C. and Josenhans, H.W., 1990. Postglacial paleoceanography of Hudson Bay: Stratigraphic, microfaunal and palynological evidence. Canadian Journal of Earth Sciences, 27: 946-963.

Church, M. and Ryder, J.N., 1972. Paraglacial sedimentation: A consideration of fluvial processes conditioned by glaciation. Geological Society of America Bulletin, 83: 3059-3072. 
Cowan, E.A. and Powell, R.D., 1990. Suspended sediment transport and deposition of cyclically interlaminated sediment in a temperate glacial fjord, Alaska, U.S.A., p. 75-89. In J.A. Dowdeswell and J.D. Scourse, ed., Glacimarine Environments: Processes and Sediments. Geological Society, London, Special Publication 53, $423 \mathrm{p}$.

Dionne, J.-C., 1978. Les champs de blocs en Jamésie, Québec subarctique. Géographie physique et Quaternaire, 32: 119-144.

Elverhøi, A., Liestøl, O. and Nagy, J., 1980. Glacial erosion, sedimentation and microfauna in the inner part of Kongsfjorden, Spitsbergen. Norsk Polarinstitutt Skrifter, 172: 33-60.

Elverhøi, A., Lønne, Ø. and Seland, R., 1983. Glaciomarine sedimentation in a modern fjord environment, Spitsbergen. Polar Research, 1: 127-149.

Eyles, N., Eyles, C.H. and Miall, A.D., 1983. Lithofacies types and vertical profile models: An alternative approach to the description and environmenta interpretation of glacial diamict and diamictite sequences. Sedimentology, 30: 393-410.

Filion, L., Saint-Laurent, D., Despontsand, M. and Payette, S., 1991. The late Holocene record of aeolian and fire activity in northern Québec, Canada. The Holocene, 1: 201-208.

Gonthier, N., d'Anglejan, B. and Josenhans, H., 1993. Seismo-stratigraphy and sedimentology of Holocene sediments off Grande Rivière de la Baleine, southeastern Hudson Bay, Québec. Géographie physique et Quaternaire, 47: 147-166.

Görlich, K., 1986. Glacimarine sedimentation of muds in Hornsund Fjord, Spitsbergen. Annales Societatis Geologorum Poloniae, 56: 433-477.

Gray, J.T., Lauriol, B., Bruneau, D. and Ricard, J., 1993. Postglacial emergence of Ungava Peninsula, and its relationship to glacial history. Canadian Journal of Earth Sciences, 30: 1676-1696.

Hill, P.R., Simard, A. and Héquette, A. 1999. High-resolution seismic stratigraphy of late Quaternary deposits in Manitounuk Sound, northern Québec: Effects of rapid post-glacial emergence. Canadian Journal of Earth Sciences, 36: 549-563.

Hillaire-Marcel, C., 1976. La déglaciation et le relèvement isostatique sur la côte est de la baie d'Hudson. Cahiers de Géographie de Québec, 20: 185-220.

Hillaire-Marcel, C., Occhietti, S. and Vincent, J.-S., 1981. Sakami moraine, Québec: A 500 km-long moraine without climatic control. Geology, 9: 210-214.

Hugues, T., 1987. Ice dynamics and deglaciation models when ice sheets collapsed, p. 183-220. In W.F. Ruddiman and H.E. Wright, Jr., ed., North America and adjacent oceans during the last deglaciation. The Geology of North America, K-3, Geological Society of America, Boulder, 501 p.

Josenhans, H.W. and Zevenhuizen, J., 1990. Dynamics of the Laurentide Ice Sheet in Hudson Bay, Canada. Marine Geology, 92: 1-26.

Josenhans, H.W., Balzer, S., Henderson, P., Nielson, E., Thorliefson, H. and Zevenhuizen, J., 1988. Preliminary seismostratigraphic and geomorphic interpretations of the Quaternary sediments of Hudson Bay. Current Research Part B: Eastern and Atlantic Canada, Geological Survey of Canada, Paper 88-1B: 271-286.

Lajeunesse, P., 2000. Géomorphologie et géologie du Quaternaire de la région, de la rivière Nastapoka, côte est de la Baie d'Hudson. Ph.D. thesis, Université Laval, $239 \mathrm{p}$.

Lajeunesse, P. and Allard, M., 2002. Sedimentology of an ice-contact glaciomarine fan complex, Nastapoka Hills, eastern Hudson Bay, northern Québec. Sedimentary Geology, 152: 201-220.

2003 The Nastapoka drift belt, eastern Hudson Bay: Implications for a stillstand of the Québec-Labrador ice margin in the Tyrrell Sea at 8 ka BP. Canadian Journal of Earth Sciences, 40: 65-76.

Lavoie, C., 2000. Formation et évolution d'un delta sur une côte en émersion, Rivière Nastapoka, Québec subarctique. M.Sc. thesis, Université Laval, Québec, $139 \mathrm{p}$

Lavoie, C., Allard, M., and Hill, P.R., 2002. Holocene deltaic sedimentation along an emerging coast: Nastapoka River delta, eastern Hudson Bay, Québec. Canadian Journal of Earth Sciences, 39: 505-518.

Lévesque, R., Allard, M. and Seguin, M.K., 1988. Le pergélisol dans les formations quaternaires de la région des rivières Nastapoka et Sheldrake, Québec nordique. Centre d'études nordiques, Université Laval, Québec, Nordicana $51,23 \mathrm{p}$.
McCabe, A.M., Bowen, D.Q. and Penney, D.N., 1993. Glaciomarine facies from the western sector of the last British Ice Sheet, Malin Beg, County Donegal, Ireland. Quaternary Science Reviews, 12: 35-45.

Meier, M.F. and Post, A., 1987. Fast tidewater glaciers. Journal of Geophysical Research, 92 (B9): 9051-9058.

Ménard, É., in prep. Cartographie et modélisation spatiale du régime thermique des sols en zone de pergélisol discontinu (Québec subarctique). Thèse de doctorat, Université Laval.

Mitrovica, J.X., 1997. Going halves over Hudson Bay. Nature, 390: 444-447.

Ó Cofaigh, C., 1998. Geomorphic and sedimentary signatures of early Holocene deglaciation in High Arctic fiords, Ellesmere Island, Canada: Implications for deglacial ice dynamics and thermal regime. Canadian Journal of Earth Sciences, 35: 437-452.

Parent, M. and Paradis, S.J., 1994. Géologie des formations superficielles, région de la Petite-Rivière-de-la-Baleine, Québec nordique. Geological Survey of Canada, Ottawa, Open File 2643, 48 p.

Parent, M., Paradis, S.J. and Boisvert, É., 1995. Ice-flow patterns and glacial transport in the eastern Hudson Bay region: Implications for the late Quaternary dynamic of the Laurentide Ice Sheet. Canadian Journal of Earth Sciences, 32: 2057-2070.

Payette, S., 1983. The forest tundra and present tree-lines of the northern Québec-Labrador peninsula, p. 3-24. In P Morissette and S. Payette, ed., Tree-line Ecology. Proceedings of the Northern Québec Tree-line Conference (Kuujjuarapik, 1981), Centre d'études nordiques, Université Laval, Québec, Nordicana 47,188 p.

Powell, R.D., 1981. A model for sedimentation by tidewater glaciers. Annals of Glaciology, 2: 129-134.

1983. Glacial marine sedimentation processes and lithofacies of temperate tidewater glaciers, Glacier Bay, Alaska, p. 185-232. In B.F. Molnia, ed., Glacial Marine Sedimentation. Plenum Press, New York, 844 p.

Saint-Laurent, D. and Filion, L., 1992. Interprétation paléoécologique des dunes à la limite des arbres, secteur nord-est de la mer d'Hudson, Québec. Géographie physique et Quaternaire, 46: 209-220.

Seramur, K.C, Powell, R.D. and Carlson, P.R., 1997. Evaluation of conditions along the grounding line of temperate marine glaciers: an example from Muir Inlet, Glacier Bay, Alaska. Marine Geology, 140: 307--327.

Simons, M. and Hager, B.H., 1997. Localization of the gravity field and the signature of glacial rebound. Nature, 390: 500-504.

Stevens, R., 1990. Proximal and distal glacimarine deposits in southwestern Sweden: contrasts in sedimentation, p. 307-316. In J.A. Dowdeswell and J.D. Scourse, ed., Glacimarine Environments: Processes and Sediments. Geological Society, London, Special Publication 53, 423 p.

Stevenson, I.M., 1968. A Geological Reconnaissance of Leaf River Map-Area, New-Québec and Northwest Territories. Geological Survey of Canada, Ottawa, Memoir 356, $112 \mathrm{p}$.

Stewart, T.G., 1991. Glacial marine sedimentation from tidewater glaciers in the Canadian High Arctic, p. 95-105. In J.B. Anderson and G.M Ashley, ed., Glacial Marine Sedimentation: Paleoclimatic Significance. Geological Society of America, Boulder, Special Paper 261, $232 \mathrm{p}$.

Stuiver, M. and Reimer, P.J., 1993. Extended ${ }^{14} \mathrm{C}$ data base and revised CALIB $3.0{ }^{14} \mathrm{C}$ calibration program. Radiocarbon, 35: 215-230.

Syvitski, J.P.M., 1991. Towards an understanding of sediment deposition on glaciated continental shelves. Continental Shelf Research, 11: 897-937.

1993. Glacimarine environments in Canada: An overview. Canadian Journal of Earth Sciences, 30: 354-371.

Syvitski, J.P.M., Burrell, D.C. and Skei, J.M., 1987. Fjords: Processes and Products. Springer-Verlag, New York, $379 \mathrm{p}$.

Vincent, J.-S. and Hardy, L., 1977. L'évolution et l'extension des lacs glaciaires Barlow et Ojibway en territoire québécois. Géographie physique et Quaternaire, 33: 357-372.

Zevenhuizen, J., 1996. Late Quaternary and Surficial Geology of Southeastern Hudson Bay. M.Sc. thesis, Dalhousie University, 215 p. 